\title{
Experimental study of aortic flow in the ascending aorta via Particle Tracking Velocimetry
}

\author{
Journal Article \\ Author(s): \\ Gülan, Utku; Lüthi, Beat; Holzner, Markus; Liberzon, Alex; Tsinober, Arkady; Kinzelbach, Wolfgang \\ Publication date: \\ 2012 \\ Permanent link: \\ https://doi.org/10.3929/ethz-b-000058740 \\ Rights / license: \\ In Copyright - Non-Commercial Use Permitted \\ Originally published in: \\ Experiments in Fluids 53(5), https://doi.org/10.1007/s00348-012-1371-8
}




\title{
Experimental study of aortic flow in the ascending aorta via Particle Tracking Velocimetry
}

\author{
Utku Gülan · Beat Lüthi • Markus Holzner • \\ Alex Liberzon • Arkady Tsinober • \\ Wolfgang Kinzelbach
}

Received: 1 June 2011/Revised: 21 July 2012/Accepted: 14 August 2012/Published online: 2 September 2012

(C) Springer-Verlag 2012

\begin{abstract}
A three-dimensional, pulsatile flow in a realistic phantom of a human ascending aorta with compliant walls is investigated in vitro. Three-Dimensional Particle Tracking Velocimetry (3D-PTV), an image-based, nonintrusive measuring method is used to analyze the aortic flow. The flow velocities and the turbulent fluctuations are determined. The velocity profile at the inlet of the ascending aorta is relatively flat with a skewed profile toward the inner aortic wall in the early systole. In the diastolic phase, a bidirectional flow is observed with a pronounced retrograde flow developing along the inner aortic wall, whereas the antegrade flow migrates toward the outer wall of the aorta. The spatial and temporal evolution of the vorticity field shows that the vortices begin developing along the inner wall during the deceleration phase and attenuate in the diastolic phase. The change in the cross-sectional area is more distinct distal to the inlet cross section. The mean kinetic energy is maximal in the peak systole, whereas the turbulent kinetic energy increases in the deceleration phase and reaches a maximum in the beginning of the diastolic phase. Finally, in a Lagrangian analysis, the temporal evolution of particle dispersion was studied. It shows that the dispersion is higher in the
\end{abstract}

U. Gülan $(\bowtie) \cdot$ B. Lüthi · M. Holzner · W. Kinzelbach

Institute of Environmental Engineering, ETH Zürich,

8093 Zürich, Switzerland

e-mail: guelan@ifu.baug.ethz.ch

A. Liberzon

Turbulence Structure Laboratory, School of Mechanical

Engineering, Tel Aviv University, 69978 Ramat Aviv, Israel

A. Tsinober

School of Mechanical Engineering, Faculty of Engineering,

Tel Aviv University, 69978 Tel Aviv, Israel deceleration phase and in the beginning of the diastole, whereas in systole, it is smaller but non-negligible.

\section{Introduction}

Hemodynamics, the study of blood flow, has become a very important research topic as the blood flow characteristics play a crucial role in cardiovascular diseases. Many hemodynamic factors influence blood flow. The arteries adapt to varying flow and pressure conditions by expanding and contracting to fulfill altering hemodynamic demands (Ku 1997). These varying hemodynamic demands can give rise to shear stresses, vortex and separation zones, secondary, rotational and disturbed flows. The aorta is the largest artery in the cardiovascular system, and the varying hemodynamic demands can be distinctive there. The aorta has a higher distensibility compared to the other arteries. It expands during the systole, when the heart pumps out blood into the ascending aorta passing through the heart valves to store about $50 \%$ of the left ventricle stroke volume (Belz 1995). It contracts in diastole, when the heart is in rest mode, to eject the stored volume. The flow in the aorta is pulsatile and three-dimensional. The complex geometry, pulsatility, three-dimensionality and the nonstationary boundaries in each pulse are important factors affecting the flow. There have been many studies on the aortic flow up to present. Segadal and Matre (1987) studied the velocity distribution in the human ascending aorta. They observed a bidirectional velocity profile in late systole and early diastole with retrograde velocities along the inner wall and a sustained antegrade flow along the outer wall (Segadal and Matre 1987). Chadran (1993) found that in systole, the axial velocity profiles at different cross sections exhibit relatively flat shapes with a skew toward 
the inner wall of curvature. In diastole, a reverse flow develops along the inner wall with an antegrade flow along the outer wall of curvature (Chandran 1993). Kilner et al. (1993) studied the human aortic arch by using threedimensional magnetic resonance velocity mapping and concluded that the helical and retrograde flows play an important role in the circulatory dynamics and pathogenesis of atheroma in the arch. They observed that the highest axial velocity occurs close to the inner curvature in early systole and a helical flow starts developing as the highest velocity stream shifts outwards. The zone where the flow separates from the inner wall is filled by retrograde flow. In the late systole and early diastole, the helical and secondary flow patterns persist (Kilner et al. 1993). Morbiducci et al. (2011) studied helical patterns in the human aorta in vivo. They observed that the highest streamwise flow velocities appear close to the inner curve and the highest velocity migrates toward the outward wall. Further, a retrograde flow develops from the relatively slow flow close to the inner curvature. They did not detect any formation of helical vortices in the ascending aorta and the proximal aortic arch in the early systole. In the peak systole, however, helical structures started to develop at the inner wall and persisted in the upper arch. In the deceleration phase, the fluid rotational momentum becomes more dominant exhibiting coherent helical and bi-helical patterns in the ascending aorta as the inertial effects provoke a disorganization of the flow field (Morbiducci et al. 2011). Stamatopoulos et al. (2011) studied the swirling flow in an abdominal aortic aneurysm model and observed that the flow develops a clockwise swirling motion in the aneurysm before peak systole and pronounced vortices are produced during the deceleration phase.

The existence of turbulence in aortas has not been completely proven. Disturbed flow is believed to be one of the key factors for cardiovascular diseases. It is assumed that the flow becomes turbulent in large arteries such as aortas (Nerem et al. 1972). McDonald (1952) studied the existence of turbulent flow in a rabbit aorta. He showed that the laminar flow becomes turbulent before the peak systole. The flow at the bifurcation of the aorta was also analyzed, and the same author reported that eddying motion occurs when there is back flow (McDonald 1952). Nerem et al. (1972) examined the velocity distribution and transition to turbulence in the thoracic aorta of dogs based on visual observation of instantaneous velocity wave forms and frequency spectrum analysis. They reported that the disturbances are confined to the systolic phase and are damped out during the diastolic phase. It was stated that the disturbances in the descending aorta are not advected from the heart but locally generated. The presence of disturbed wave forms was not only related to the peak Reynolds number (Nerem et al. 1972). Stein and Sabbah (1976) investigated turbulent blood flow in the ascending aorta of humans. It was shown that disturbed flow occurs in the ascending aorta of healthy subjects and develops at peak velocity. Turbulent flow was observed in the ascending aorta of subjects with aortic valvular disease, with high turbulent energy densities in aortic stenosis in particular. In these patients, disturbances occur before peak systole and extend along the diastolic phase (Stein and Sabbah 1976). Yamaguchi et al. (1987) studied aortic flow through nonstationary spectral analysis of turbulence. They concluded that the temporal variation of turbulence characteristics in the aorta is rapid and elevated turbulence intensity commences close to peak systole and attains its maximum in the latter half of the deceleration phase and finally decays at the end of the pulse (Yamaguchi et al. 1987).

For more than two decades, PTV has been in use as a Lagrangian flow measurement technique. Over the last 15 years, (Virant and Dracos 1997; Malik et al. 1993; Maas et al. 1993; Wilneff and Gruen 2002; Kasagi and Matsunaga 1995; Hoyer et al. 2005; Lüthi et al. 2005; Holzner et al. 2008) applied 3D-PTV for a vast range of complex and usually turbulent flows. The method has also been used in biomedical applications in Schibli et al. (2008, Boutsianis et al. (2009) and Balducci et al. (2004), among others. Medical imaging techniques have been used for clinical purposes and medical research for decades. Magnetic resonance imaging (MRI), doppler ultrasound and computed tomography (CT) have been widely used as imaging tools. Despite continuous further developments and improvements, all the techniques have limitations to access the instantaneous flow field and therefore the turbulent fluctuations and shear stresses. Recently, the 4D velocity magnetic resonance imaging has been applied in many studies to access the velocity field in vessels (Bogren et al. 2004; Dyverfeldt et al. 2006, 2008; Frydrychowicz et al. 2007; Kilner et al. 1993; Kozerke et al. 2001; Kvitting et al. 2010; Markl et al. 2005) and Morbiducci et al. (2009). One of the aims of this study is to measure both the instantaneous and the phase-averaged flow field in a realistic aorta. MRI has limitations to extract the instantaneous flow field and provides mainly information on the phaseaveraged flow field. Recent developments on phase-contrast-MRI (PC-MRI) (Dyverfeldt et al. 2006, 2008) allow to access also velocity fluctuations. The results presented may serve as a reference for turbulence information in computational studies and further developments for the PC-MRI techniques. As another drawback of MRI, the reliability of the flow velocities measured in the diastolic phase is debateable. The signal-to-noise ratio is very low as a consequence of the smaller velocity magnitudes during this time period of the flow. The present study can be used to improve the MRI velocity acquisition methods in the diastolic phase. 
A three-dimensional, pulsatile flow in an elastic and anatomically accurate aortic replica is experimentally investigated. From the applied 3D-PTV technique, we obtain both, Lagrangian and Eulerian information. As a Lagrangian study, we analyzed the flow's dispersive property and temporal evolution of Lagrangian trajectories. In addition, we present detailed Eulerian data for flux, velocity field and the phaseaveraged vorticity field as well as recordings of the fluctuative part of the flow. Our findings indicate that the fluctuative part of the velocity field becomes significant, particularly during the deceleration phase of the flow.

\section{Experimental setup}

The aortic flow is in essence complex due to pulsatility, threedimensionality and the non-steady-state boundaries under the effect of compliance. To perform realistic in vitro measurements, it is crucial to imitate, at least qualitatively, the relevant in vivo conditions. The experimental setup comprises mainly the aortic phantom, a ventricular assist device (VAD) to mimic the function of the heart, a pump system to drive the VAD and the optical part as shown in Fig. 1.

The measurements were performed in a realistic aortic phantom manufactured by Elastrat (Geneva, Switzerland) reconstructed from a three-dimensional volume rendering of a high-resolution MRI scan, resembling the vascular geometry of a healthy patient. The inlet diameter of the aorta is $d=21 \mathrm{~mm}$. The aorta replica consists of three main parts including an ascending aorta, an aortic arch and a descending aorta. A ventricular assist device (VAD) is a mechanical pump mimicking the function of the heart. VADs provide a good solution for people having a heart failure problem and for people waiting for a heart implant. In the measurements, a VAD produced by MEDOS (Stolberg, Germany), with a pump chamber of $54 \mathrm{ml}$ was used to pump the working fluid into the aortic phantom. To drive the flow, a custom designed PWG-01-ST pressure and vacuum pump by Innovative Technology and Engineering Solutions (Haifa, Israel) was used.

The optical part comprises a laser, a high-speed camera, an image splitter and an orange filter. A BeamLok 2080 (Spectra Physics) laser was used to illuminate the investigation domain. The BeamLok 2080 is a $25 \mathrm{~W}$ continuous laser with a wavelength of $514 \mathrm{~nm}$. The laser path was guided by mirrors. A laser volume with a thickness of $3 \mathrm{~cm}$ was generated by using different lenses. A Photron SA5 (Tokyo, Japan) camera was used to acquire images with high temporal resolution. The high-speed camera allows to record $1.56 \mathrm{~s}$ at full resolution of $1024 \times 1024$ pixels and 7,000 fps with 16GB memory. A 12-bit analog digital converter (ADC) (Bayer system color, single sensor) with $20 \mu \mathrm{m}$ pixel sensor provides higher light sensitivity for high-speed recordings. A Nikon AF Micro-Nikkor $60 \mathrm{~mm}$

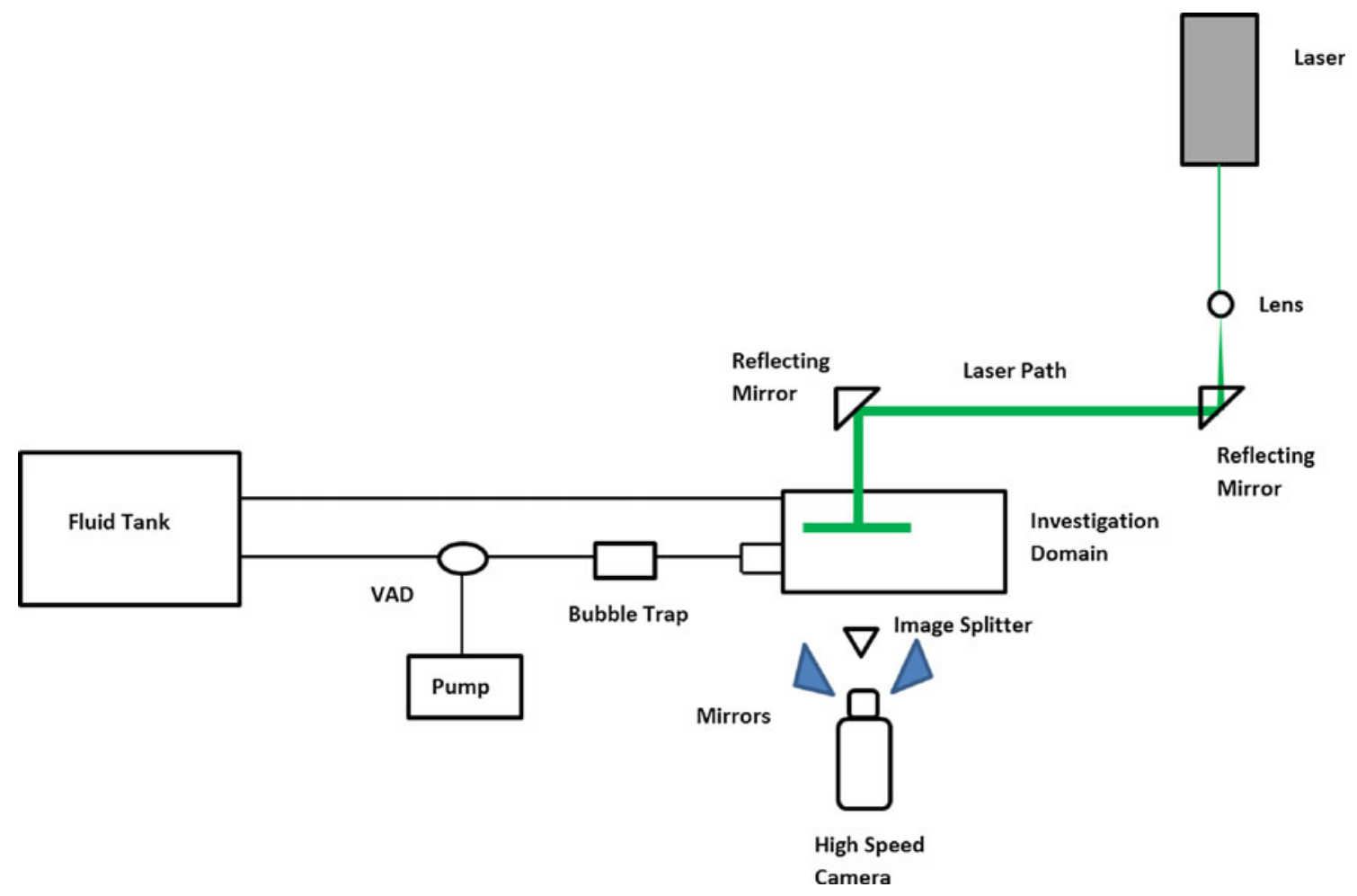

Fig. 1 Schematic view of the experiment setup 


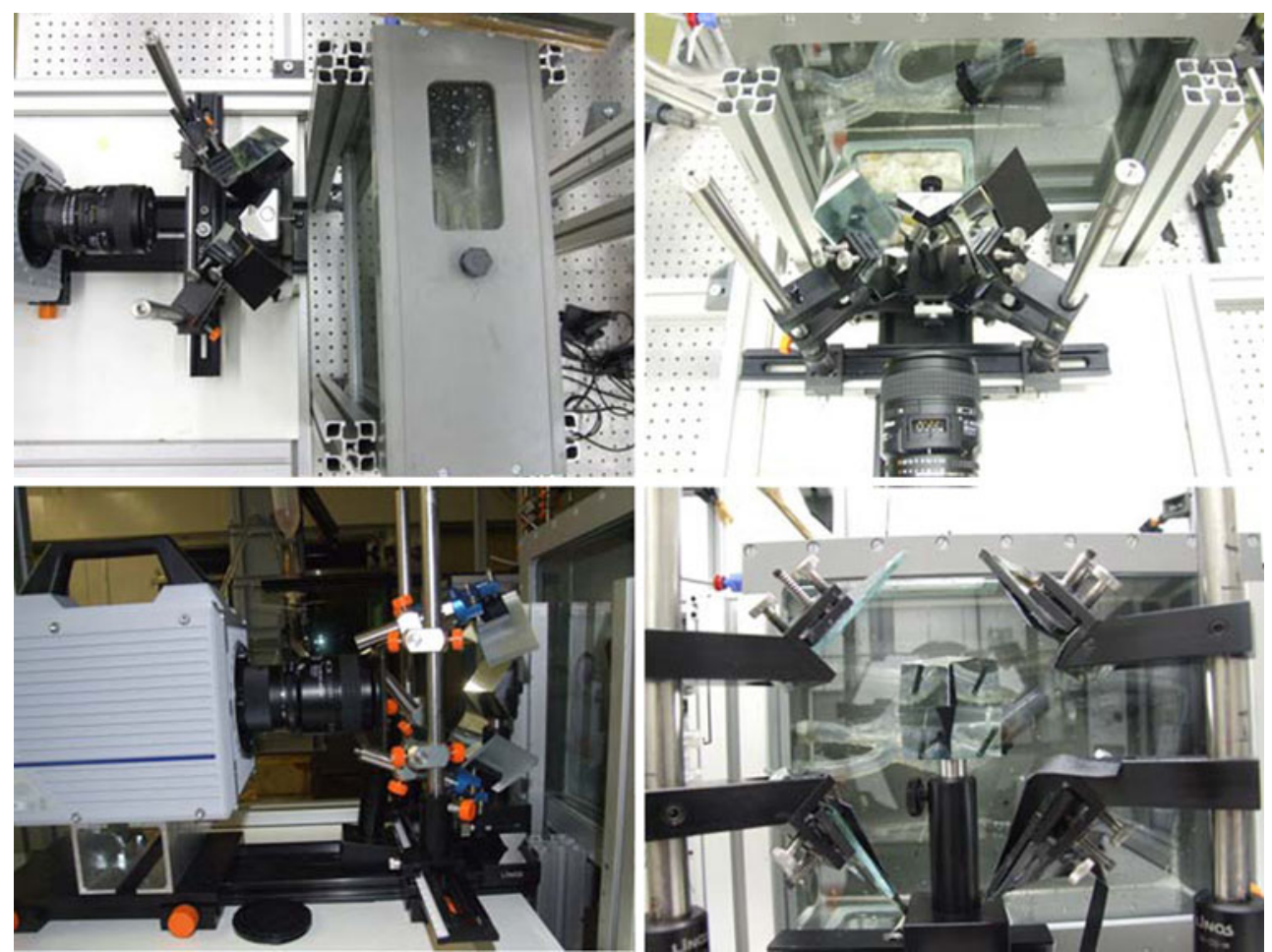

Fig. 2 Photographs of the optical setup showing the high-speed camera with image splitter

f/2.8D lens was used. The camera was synchronized with the pump system to trigger the recordings at the beginning of the pulse wave. Figure 2 depicts the optical setup including the camera, mirrors and an image splitter for four different views. The image splitter system was used to mimic four different views by using a single camera as shown in the figure. The four-way splitter consists of a set of four fixed primary slanted mirrors assembled on a regular pyramid and four secondary mirrors (Hoyer et al. 2005). As shown in the front view of the optical setup, the alignments of the mirrors play an important role in the stereoscopic imaging. The optimum arrangement of the mirrors is such that the acquired images for all four mirrors cover the same domain at an angle of around $45^{\circ}$ and at comparable distances, such that all four views are within the focused distance set by the lens.

3D-PTV is capable to work in an environment with multiple indexes of refraction, as long as the interfaces are planar. Since the aorta replica has an arbitrary geometry, this implies that the working fluid needs to have the same index of refraction as the silicon used. In our measurements, the optical path comprises air-glass-fluid-silicon-fluid from the camera toward the investigation domain. For this purpose, the index of refraction of silicon and the working fluid should be identical to minimize the optical distortions. Figure 3 displays the quality of the matching of index of refraction of the silicon medium for different working fluids.

In Fig. 3a, air with a refractive index of $n=1$ was used as a working fluid. It is apparent that the dots on the calibration target behind the silicon block are indiscernible. In Fig. 3b, water with a refractive index of $n=1.33$ was chosen as a working fluid. The visibility of the dots on the calibration target is relatively better compared to air. Particularly, along the boundaries and the curvatures, the effect of the refractive index mismatch is still significant. The discontinuity along the curvatures over the whole domain is more distinct as a consequence of the optical distortions. Finally, Fig. 3c depicts the matching of the refractive indexes of silicon and a mixture of glycerin, water and $\mathrm{NaCl}$. Since the index of refraction of both silicon and the mixture is 1.41, the optical disturbances become comparatively negligible over the domain. As an additional constraint, the working fluid should also match a typical kinematic viscosity of blood. Blake et al. (2009) proposed a new mixture of fluid feasible for image-based measurements. A mixture of $37 \%$ glycerol, $48 \%$ water and $15 \%$ sodium chloride with a refractive index of 1.41 and kinematic viscosity of $4.85 \times 10^{-6} \mathrm{~m}^{2} / \mathrm{s}$ is used as working fluid in our study.

Choosing the optimal tracers is significant for the 3D-PTV technique. Depending on the dimensions of the investigation volume, the optimal diameter of the particles may vary. Particles should be big enough to be detected in the image processing, that is, they should cover a few pixels at least, and small enough to follow the flow reliably, that is, the Stokes number should be well below unity. In our measurements, fluorescent rhodamine particles with a 

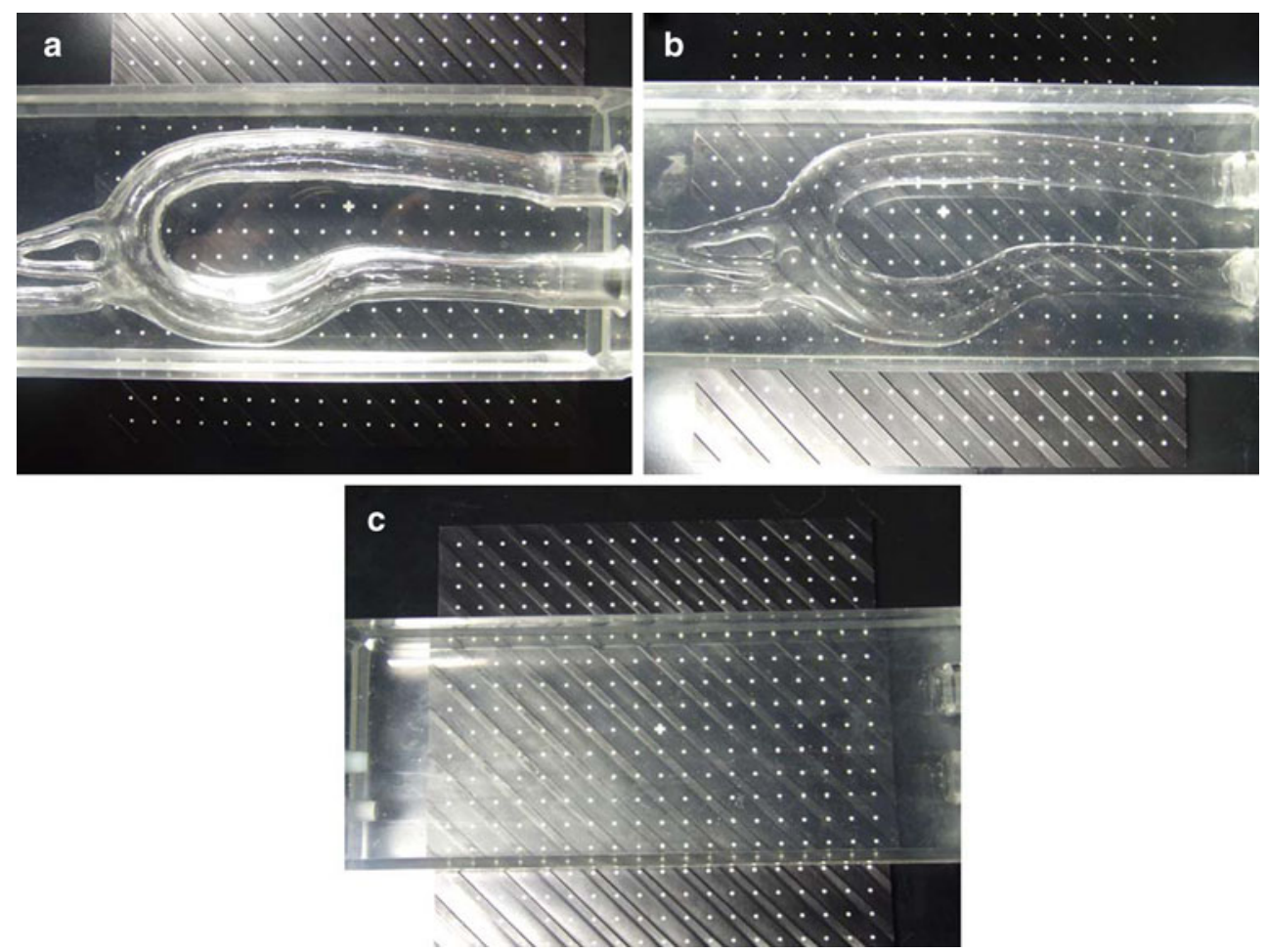

Fig. 3 Matching of index of refractions for different working fluids. air (a), water (b), glycerin +water+NaCl mixture (c)

diameter range of $180-200 \mu \mathrm{m}$ were used as particles. The resulting Stokes number, St, thus is $O(0.01-0.001)$, with $S t=\tau_{p} / \tau_{f}$ and for neutrally buoyant particles $\tau_{p}=d_{p}^{2} /$ $(18 v) \sim 5 e-4$. The flow time scale can be assumed as $\tau_{f} \sim$ $0.1-0.01 \mathrm{sec}$. This means that the particles follow the flow faithfully. The fluorescent tracers used in this study are not commercial products. The tracer particles were produced based on the study of Pedocchi et al. (2008). The particle density is estimated at $1.2 \mathrm{~g} / \mathrm{cm}^{3}$ (Pedocchi et al. 2008). The excitation and emission wavelengths of rhodamine wt are 558 and $582 \mathrm{~nm}$, respectively (Wilson et al. 1986). Illuminated particles are excited at a certain wavelength and emit fluorescent light. As the investigation domain comprises unsteady boundaries and an irregular volume, undesired optical noise may arise during the illumination. To avoid the optical noise such as refraction, an optical filter can be used. In our measurements, an orange bandpass filter with a wavelength of $514 \mathrm{~nm}$ was used to block the optical noise. The dimensions of the investigation domain are $50 \mathrm{~mm} \times 25 \mathrm{~mm} \times 25 \mathrm{~mm}$. The number of the particles seen by all four cameras is not constant along the pulse as a consequence of the non-steady-state boundaries. As the aorta expands in the systolic phase and shrinks in the diastolic phase, the total number of particles over the entire domain varies in time. The average number of particles is around 500 for all cameras. The results presented in this study are phase-averaged results unless specifically stated. The phase-averaged data consist of 42 different measurements, for each of which 8,000 corresponding image frames are analyzed. The Eulerian results are obtained by transforming the Lagrangian information into a Eulerian grid for all measurements. The Eulerian grid consists of $34 \times 20 \times 25$ voxels over the entire investigation domain with dimensions $2 \mathrm{~mm} \times 2 \mathrm{~mm} \times$ $2 \mathrm{~mm}$. The average number of particles in a single voxel after the phase averaging process is 155 . The particle density can be calculated as $155 /(2 \times 2 \times 2)=19$ particles $/ \mathrm{mm}^{3}$.

Calibration, that is, the determination of external and internal camera parameters, such as position, orientation, focal distance, optical axis correction, plays a significant role in obtaining an accurate result. To constitute the correspondences between images, it is crucial to obtain the particle position in real space with a high precision. A higher quality calibration leads to reduced ambiguities. The calibration can be performed statically or dynamically. Depending on the available space and accessibility of the investigation domain, it can be difficult to position a static calibration target in the domain. To overcome this problem, the dynamic calibration or also called "dumbbell calibration" is an effective tool. In contrast to the statical calibration target, the dumbbell calibration target comprises two points with a well-defined distance (Fig. 4 upper). The main principle is to move the calibration target around the investigation domain. One of the advantages of the dumbbell calibration is that the correspondences are 
Fig. 4 Dumbbell calibration target (upper) and tracking of the target in time (lower)
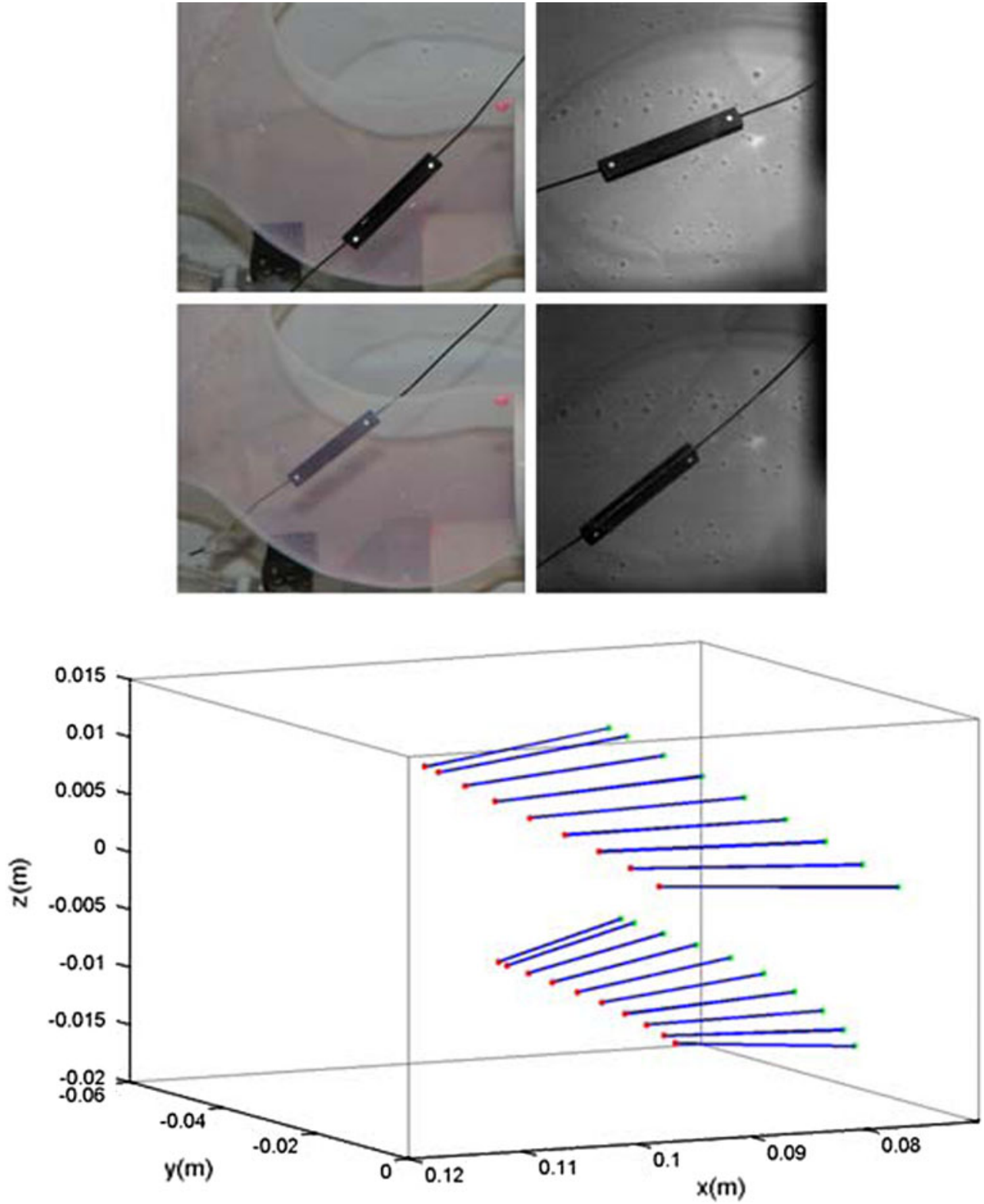

established by virtue of a rough initial guess. Two points are used to access the epipolar lines. Starting from the initial guess, the calibration optimizes the distances between the points as a consequence of the misaligned epipolar lines. Another difference from the static calibration is that the dynamic calibration uses four cameras simultaneously. The algorithm of the dynamic calibration uses the epipolar misalignment and the mismatch of the distance of two points consecutively. As the algorithm computes twelve camera positions, three camera angles, two chip positions and one local distance, the procedure is slower. The lower figure in Fig. 4 illustrates the motion of the dynamic calibration target. Red and green points describe the different points of the calibration target. As can be seen from the figure, the dumbbell calibration target is moved both in front and behind the silicon replica. As a conclusion, the camera positions are calibrated with a comparatively accurate and robust method.

The accuracy of the measurements is a significant indicator to identify the limits of the measurement technique. Although the accuracy of the 3D-PTV measurements varies from measurement to measurement, in general, the measurement accuracy of the experiments presented in this study can be estimated as follows. The position accuracy of the particles is in the range of 0.15 , $0.15,0.3 \mathrm{~mm}$ in $x, y$ and $z$ directions, respectively. The velocity uncertainty of the raw PTV data, $\varepsilon_{\mathrm{ptv}}=0.033 \mathrm{~m} / \mathrm{s}$, was obtained from the calibration. A Savitzky-Golay filter was applied for smoothing in time along Lagrangian trajectories, using a cubic polynomial fitted to 21 frames. 
The experimental accuracy can be calculated by $\varepsilon_{\mathrm{ptv}} / \sqrt{\kappa_{\text {size }} / t_{\mathrm{fps}}}$, where $\kappa_{\text {size }}$ is the filter size of $3 \mathrm{~ms}$ and $t_{\mathrm{fps}}$ is the frequency of the camera. This filtering reduces the velocity uncertainty to $0.0072 \mathrm{~m} / \mathrm{s}$. The accuracy of a velocity assigned to a voxel can be calculated as $\varepsilon_{\mathrm{ptv}} / \sqrt{n_{p} \times n_{e}}$, where $n_{p}$ is the typical number of particles in the voxel and $n_{e}$ is the number of experiments. The final voxel accuracy is around $4.09 \times 10^{-4} \mathrm{~m} / \mathrm{s}$.

Although 3D-PTV is a standard method for mapping both the Lagrangian and Eulerian information about the flow, there are some limitations. It is substantially difficult to accurately determine the boundaries for the flow in a chamber with a non-uniform shape. Basically, the method is to declare a boundary, just next to the outmost regions where moving particles are observed. For this approach, obviously, the seeding density is one of the crucial parameters affecting the determination of the boundaries. The seeding density is chosen such that the number of particles should be high enough to obtain sufficient data over the investigation domain, particularly the boundary region. Also, the seeding density should be low enough to optimize the ambiguities while establishing the correspondences. As the aortic phantom comprises non-steadystate boundaries, the compliant property of the model limits 3D-PTV measurements. To quantify this inaccuracy, a comparison between the cross-sectional areas of the measured compliant model and the calculated phantom geometry was performed. Figure 5 describes the general frame of the limitation of the 3D-Particle Tracking Velocimetry from the point of view of cross-sectional area determination. Figure 5 displays the comparison of the cross-sectional areas of measured compliant and calculated phantom models. As the geometry of the compliant model varies over the pulse, the first time step which corresponds to the beginning of the pulse is chosen as reference time instant for both models. It is seen from the figure that the maximum discrepancies between the cross-sectional areas of the measured and the calculated models reach up to

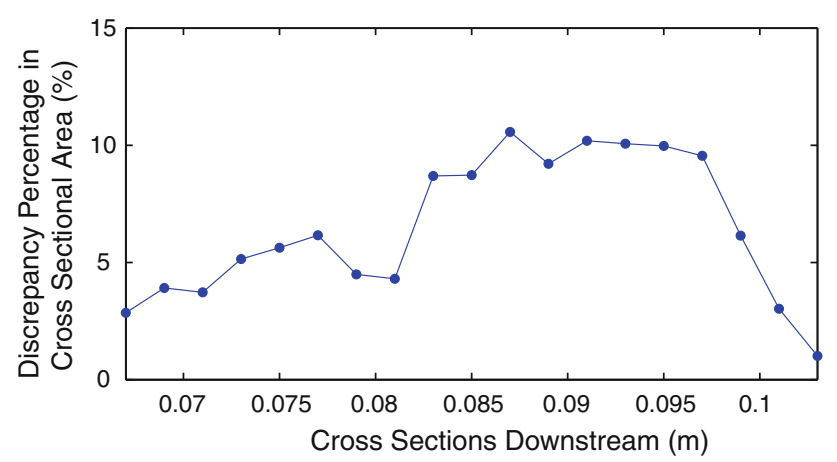

Fig. 5 Comparison of the cross-sectional areas of measured and calculated phantom model
$10 \%$. In the coordinate system used, the cross section at $0.1030 \mathrm{~m}$ is the inlet cross section and the one at $0.067 \mathrm{~m}$ is the outlet cross section along the ascending aorta. It can be seen in the figure that the minimum difference arises at the inlet cross section with a discrepancy of $1 \%$. The differences are relatively lower at the inlet cross section and the regions close to the outlet cross section of the ascending aorta. The discrepancies amplify along the midcross sections which correspond to the region where the separation zones and rotational regions develop. The detailed explanation of the flow field will be given in Sect. 3 below. The flow complexity and lack of particles passing through the boundary region are two key reasons responsible for the differences between the models. In general, it can be said that the mean error of the determination of the cross-sectional area of the measured compliant model is around $6 \%$.

\section{Results and discussion}

The aortic flow can be described as a three-dimensional, pulsatile and disturbed flow. The investigation domain covers the ascending aorta as shown in Fig. 6. Three different cross sections are displayed in the figure for later reference. For the ascending aorta measurements, 42 heartbeats were used. Each heartbeat corresponds to a single pulse with a length of $1.159 \mathrm{~s}$. The parameters used during the measurements are presented in Table 1.

The flow velocity obtained via PTV is decomposed into its mean, pulsatile and turbulent components, that is, $\mathbf{u}(\mathbf{x}, \mathbf{t})=$ $\overline{\mathbf{u}}(\mathbf{x})+\tilde{\mathbf{u}}(\mathbf{x}, t)+\mathbf{u}^{\prime}(\mathbf{x}, t)$. The phase-averaged velocity $\hat{\mathbf{u}}(\mathbf{x}, t)=\overline{\mathbf{u}}(\mathbf{x}, t)+\tilde{\mathbf{u}}(\mathbf{x}, t)$ is obtained as $\frac{1}{n} \times \sum \mathbf{u}(\mathbf{x}, t)$ where $n$ is the number of pulses. We refer to the turbulent part by $\mathbf{u}^{\prime}(\mathbf{x}, t)$. In particular, mean turbulent kinetic energy is obtained as $k(t)=\frac{1}{2 n V} \times \sum \int[\mathbf{u}(\mathbf{x}, t)-\widehat{\mathbf{u}}(\mathbf{x}, t)]^{2} \mathrm{~d} V$.

The phantom has compliant boundaries and the crosssectional area varies along the pulse. Figure 7 presents the temporal evolution of the cross-sectional area for all downstream cross sections along the ascending aorta. At the beginning of the pulse, all the cross sections have similar areas. At the inlet cross section of the ascending aorta which corresponds to cross section I in Fig. 6, the temporal variation of the cross-sectional area is small compared to the other cross sections. In the systolic phase, the cross-sectional area increases as a consequence of the higher pressure. When the flow is pumped into the ascending aorta, the aorta expands in all directions. At the peak systole, the cross-sectional areas for all cross sections reach a maximum. At that time instant, the cross-sectional area is maximal at $x=0.058 \mathrm{~m}$. In the deceleration phase, the cross-sectional areas become smaller for the entire domain. The region between outlet and mid ascending 
Fig. 6 Schematic view of the investigation domain
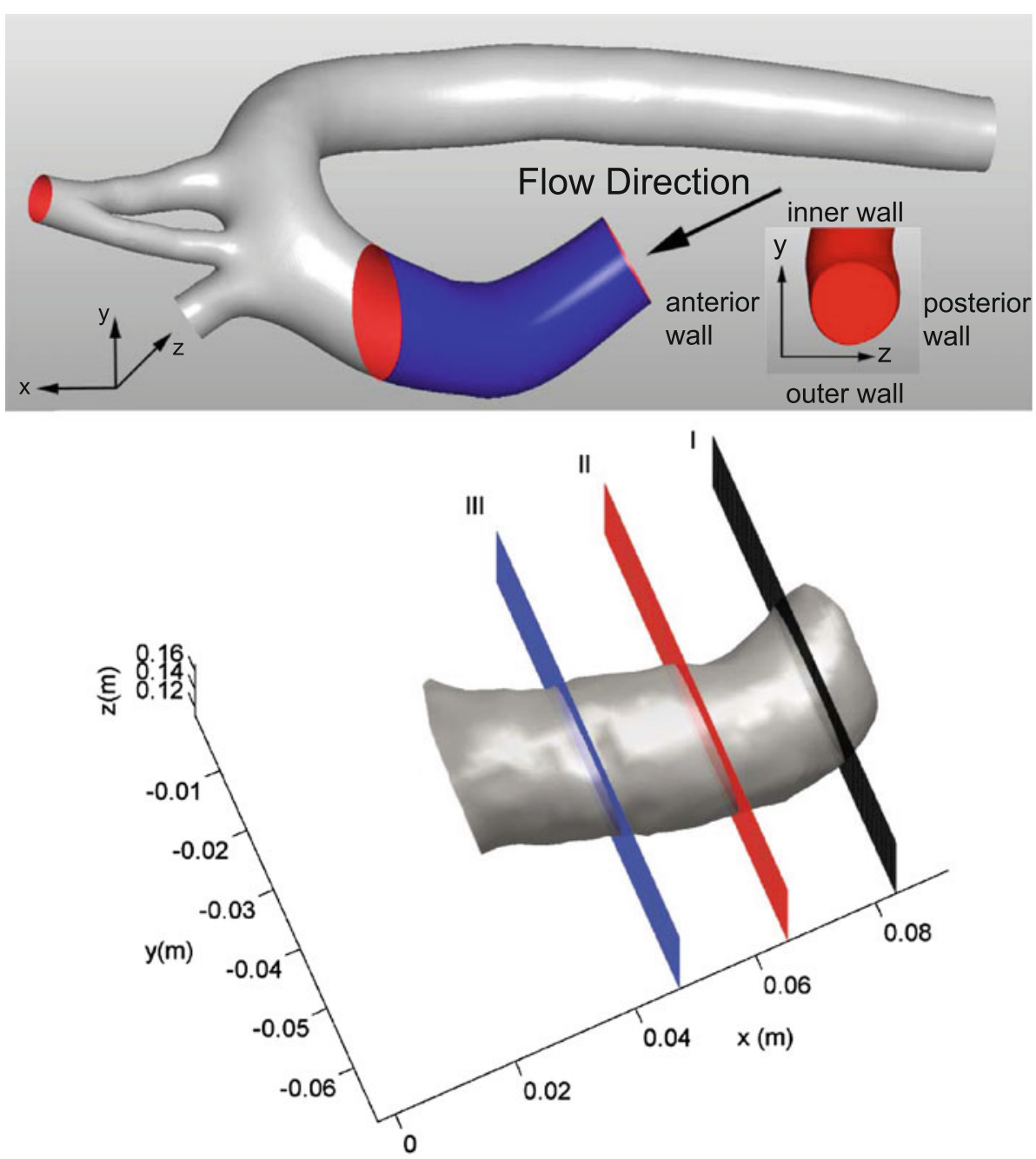

Table 1 Working fluid properties and flow parameters used in the measurements

\begin{tabular}{lll}
\hline Parameter & Unit & Variable \\
\hline Density of the fluid & $\mathrm{gr} / \mathrm{cm}^{3}$ & 1.2 \\
Kinematic viscosity of the fluid & $\mathrm{m}^{2} / \mathrm{s}$ & $4.85 \times 10^{-6}$ \\
Stroke volume & $\mathrm{ml}$ & 54 \\
Pulse duration & $\mathrm{s}$ & 1.159 \\
Investigation domain & $\mathrm{mm}^{3}$ & $50 \times 25 \times 25$ \\
Womersley number & & 11 \\
Frame rate & $\mathrm{Hz}$ & 7000 \\
Peak antegrade velocity & $\mathrm{m} / \mathrm{s}$ & 0.536 \\
Peak retrograde velocity & $\mathrm{m} / \mathrm{s}$ & -0.547 \\
Mean antegrade velocity & $\mathrm{m} / \mathrm{s}$ & 0.129 \\
Mean retrograde velocity & $\mathrm{m} / \mathrm{s}$ & -0.042 \\
Mean bidirectional velocity & $\mathrm{m} / \mathrm{s}$ & 0.096 \\
Peak Reynolds number & & 2323 \\
Mean Reynolds number & & 415 \\
Number of Pulses & & 42 \\
\hline
\end{tabular}

aorta shows a larger variation compared to the other cross sections in the diastolic phase. In the mid-diastolic phase, all cross-sectional areas are minimal. Comparing all cross sections in the mid-diastolic phases shows that the regions close to the outlet of the ascending aorta are the regions affected more dominantly by the compliance. In the diastolic phase, the aorta shrinks as a consequence of the pressure drop. Thereafter, through the late diastolic phase, all the cross sections return to the same area as in the beginning of the pulse.

To present a visual observation of the compliance during a pulse, the comparison of the geometrical deformation of the aorta for two time instants is depicted in Fig. 8. The viewpoint is rotated with respect to Fig. 6 to emphasize the region with the highest deformation.

It is apparent that the phantom geometry undergoes drastic changes over the short time interval between $t=0.51$ and $0.77 \mathrm{~s}$. In the mid-diastolic phase which corresponds to $t=0.77 \mathrm{~s}$, the deformation distal to the inlet cross section is considerable compared to the 
Fig. 7 Variation of cross-sectional areas for all downstream cross sections as a function of time

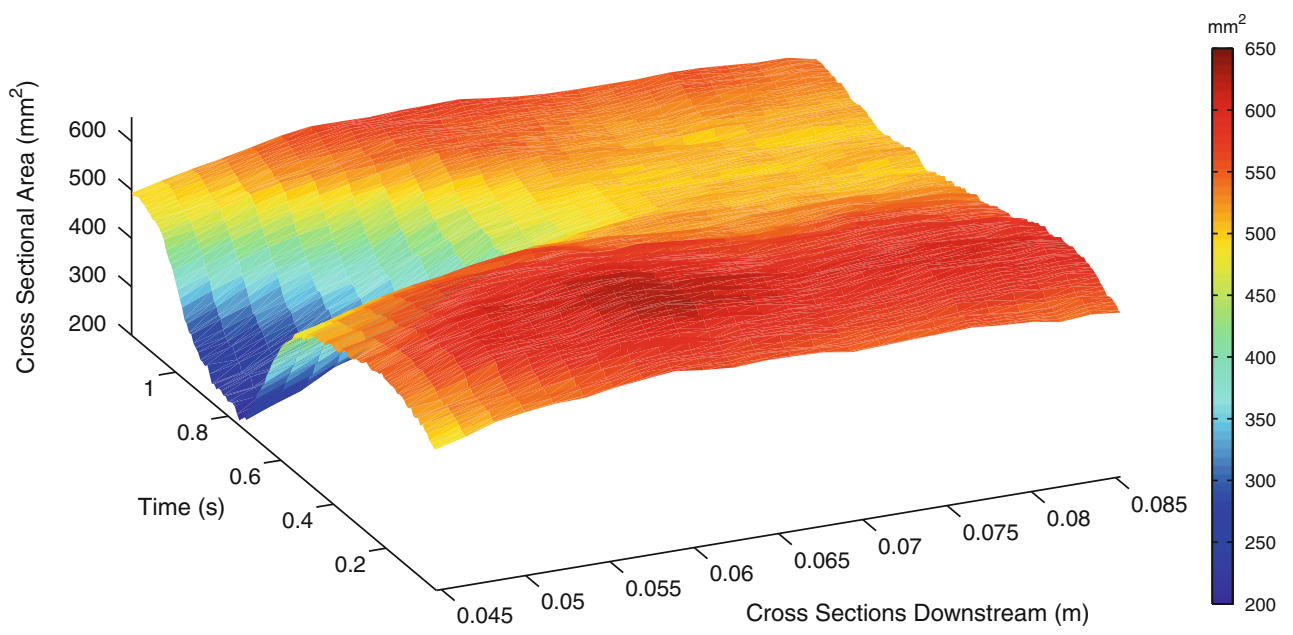

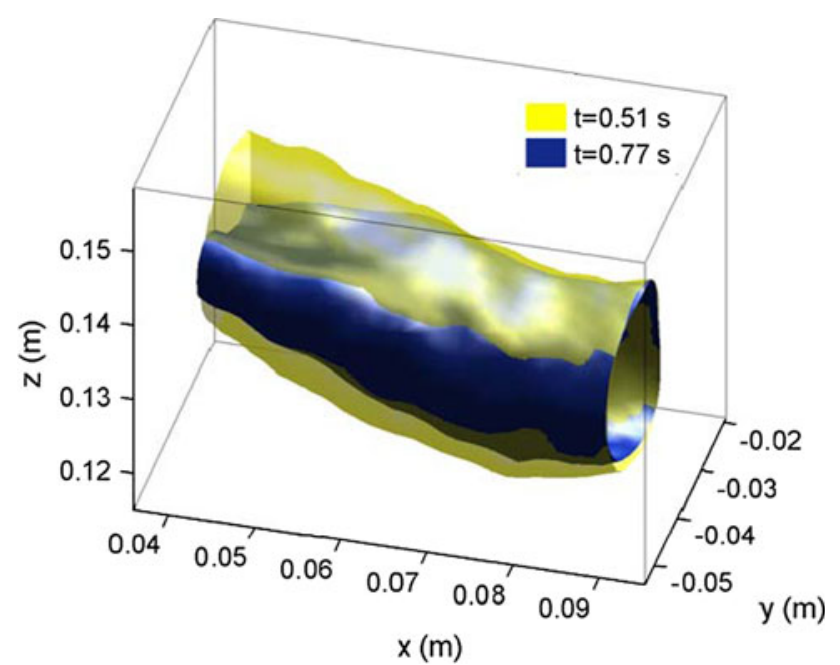

Fig. 8 Isosurface plot of aortic boundary deformation at $t=0.51$ and $t=0.77 \mathrm{~s}$

geometry in the deceleration phase which corresponds to $t=0.51 \mathrm{~s}$. As it is shown in the figure, the deformation at the outlet cross section (in the proximity of the aortic arch) is more distinctive in $z$-direction. It is also apparent that contraction of the geometry in $z$-direction is associated with a dilatation of the geometry in $y$-direction.

Figure 9 depicts the phase-averaged volumetric fluxes for different cross sections as labeled in Fig. 6. The phaseaveraged volumetric fluxes are calculated integrating the streamwise velocity over the cross-sectional area. The dashed lines refer to the mean volumetric flux for three cross sections. The mean volumetric fluxes overlap for all cross sections with magnitudes of 50.4, 50.8 and $51 \mathrm{ml} /$ pulse at outlet, mid and inlet cross sections, respectively. The difference between the mean volumetric fluxes at different cross sections shows the error of $Q(1 \%)$. It is seen from Fig. 9 that there is a period of highly positive volumetric flux $(Q>0)$ corresponding to systole, followed

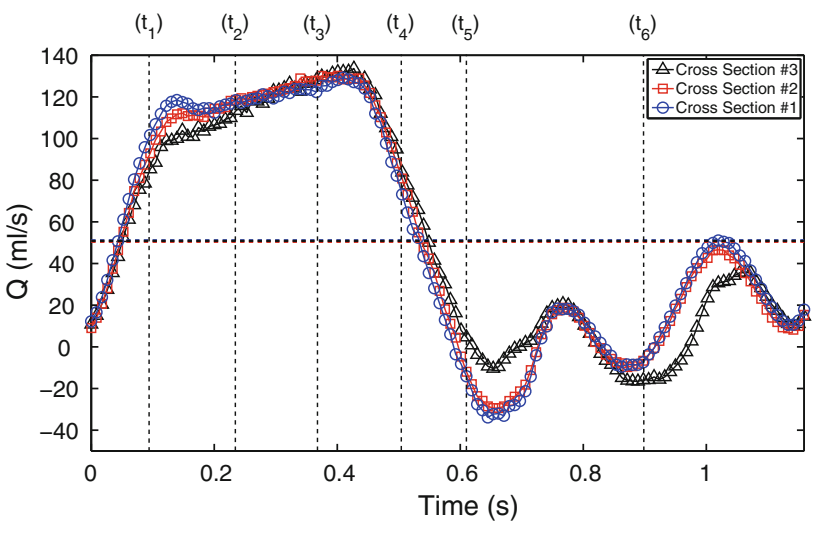

Fig. 9 Phase-averaged volumetric fluxes as a function of time for three different cross sections. The dashed lines refer to the mean volumetric flux

by an interval of lower volumetric flux (including $Q<0$ ) and an oscillating volumetric flux during diastole. In the systolic phase, there is a prominent increase from the beginning of the pulse up to the systolic peak for all cross sections. The inlet cross section has a higher magnitude of volumetric flux compared to cross sections II and III during this period. Thereafter, there is a slight increase in the plateau region for all cross sections with the inlet cross section showing lower magnitude of the volumetric flux. During the deceleration phase, the inlet cross section persists showing lower volumetric flux. In the beginning of the diastole, the volumetric fluxes reach the minima for all cross sections where a reverse flow takes place $(Q<0)$. Through the diastolic phase, the magnitudes of the phaseaveraged volumetric fluxes become smaller compared to their values in the systolic phase. Through the diastolic phase, the volumetric fluxes oscillate around magnitudes smaller than those in the systolic phase for all cross sections. The oscillations can be attributed to the Windkessel effect. As a consequence of the elasticity of the aortic 
walls, the aorta expands in the systole and stores some portion of the stroke volume. In the diastolic phase, the aorta releases the stored volume. There is a distinct discrepancy during the diastolic phase at cross section III. This is the region where the aorta has a higher contraction in diastole as depicted in Fig. 7. Consistently with the cross-sectional variation, the volumetric flux is substantially lower in the diastole.

Figure 10 displays the 3-D velocity profiles at the inlet cross section which corresponds to cross section I in Fig. 6. In the beginning of the systole $\left(t_{1}\right)$, the spatial distribution of the velocity profile is relatively uniform, slightly skewed toward the inner wall. In the peak systole $\left(t_{2}\right)$, the velocity profile is skewed toward the inner wall of the aorta. In the third time instance $\left(t_{3}\right)$, the velocity profile is similar to the previous time instants with a higher magnitude at the inner wall. In the deceleration phase $\left(t_{4}\right)$, the skewness of the profile vanishes and the velocity profile transforms into a more parabolic form. In the beginning of the diastolic phase $\left(t_{5}\right)$, the magnitudes of the velocities attenuate strongly. The velocity profile has now a more irregular pattern. The bulk of the flow comprises forward flow, whereas the reversal flow becomes visible at the boundaries along the inner wall. In the final time instant, the magnitudes of the velocities are lower compared to those during the rest of the pulse.

The temporal evolution of the velocity distribution at different cross sections is presented in Fig. 11. The streamwise velocity component is color-coded, and the inplane velocity components are displayed as vectors. In the beginning of the systolic phase, the spatial pattern of the velocity field is comparatively uniform for all cross sections, whereas the inlet cross section has a higher velocity region close to the inner wall of the aorta. The second cross section has a higher velocity zone in the bulk of the flow, and the final cross section has a higher velocity region close to the outer wall of the aorta. The approximate location of the maximum streamwise velocity region varies in time. In the second time instant, the magnitude of the velocities increases for all cross sections. In the third time instant, two counter rotational vortices start developing at cross section II (mid). Similarly, a rotational zone becomes visible at cross section III (outlet). In contrast to cross sections II and III, there is no evidence for rotational zones at cross section I (inlet). In the deceleration phase, the counter rotational regions become more apparent at cross sections II and III. At cross section III, the higher velocity region migrates toward the outer boundary of the ascending aorta. In the beginning of the diastole, which corresponds to the final time instant, a bidirectional flow develops for all cross sections, and concomitantly, the spatial pattern of the velocity field becomes more irregular for all cross sections compared to the prior time instants. At the inlet cross section, the reversal flow develops along the boundaries, whereas at cross sections II and III, it develops close to the inner wall. The counter rotational vortices are preserved at cross sections II and III.

We have seen in Fig. 11 that rotational and retrograde flow regions arise in the domain. To get a better overview over these regions, the phase-averaged velocity vector
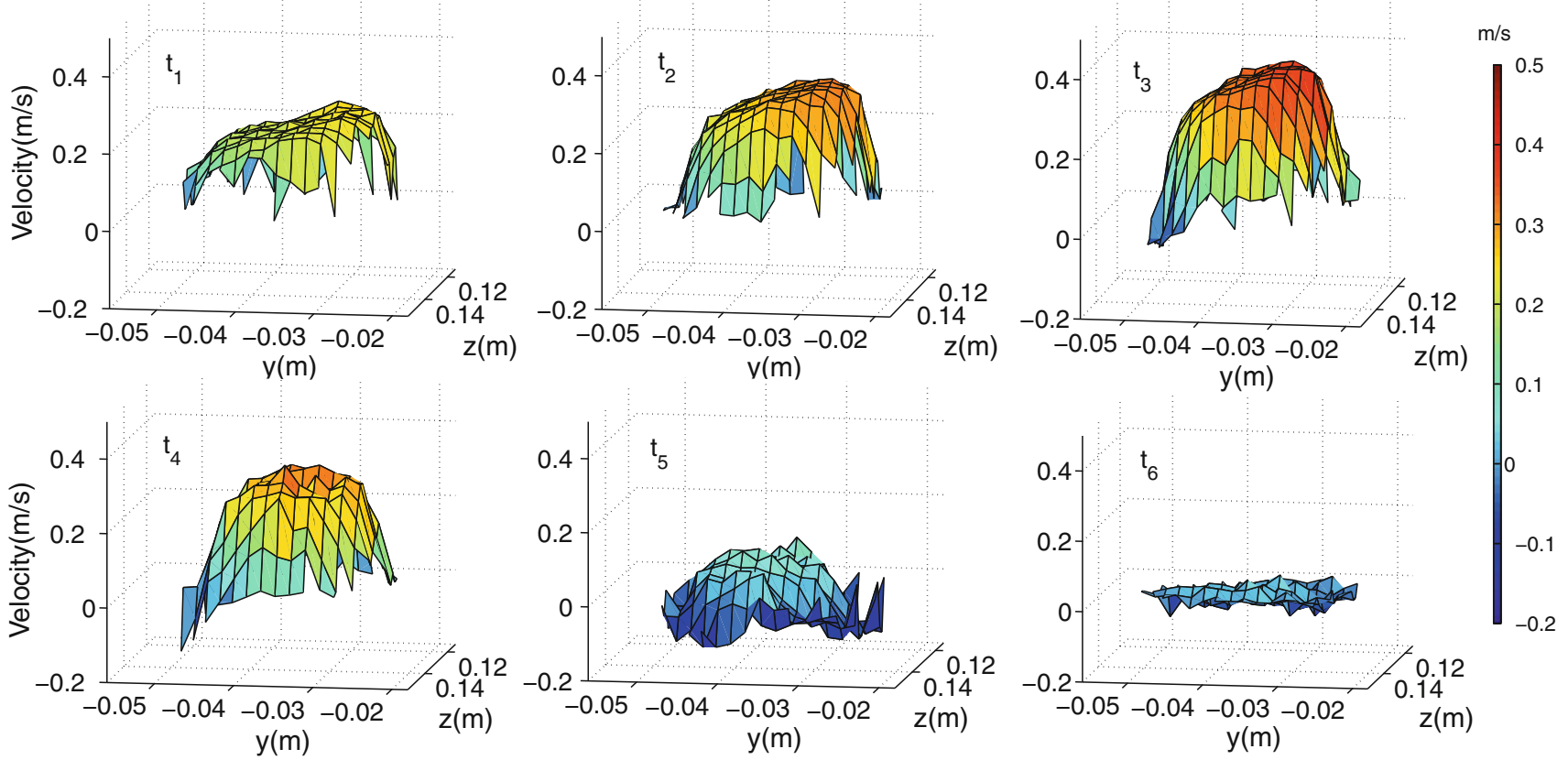

Fig. 10 Temporal evolution of velocity profiles at the inlet cross section 


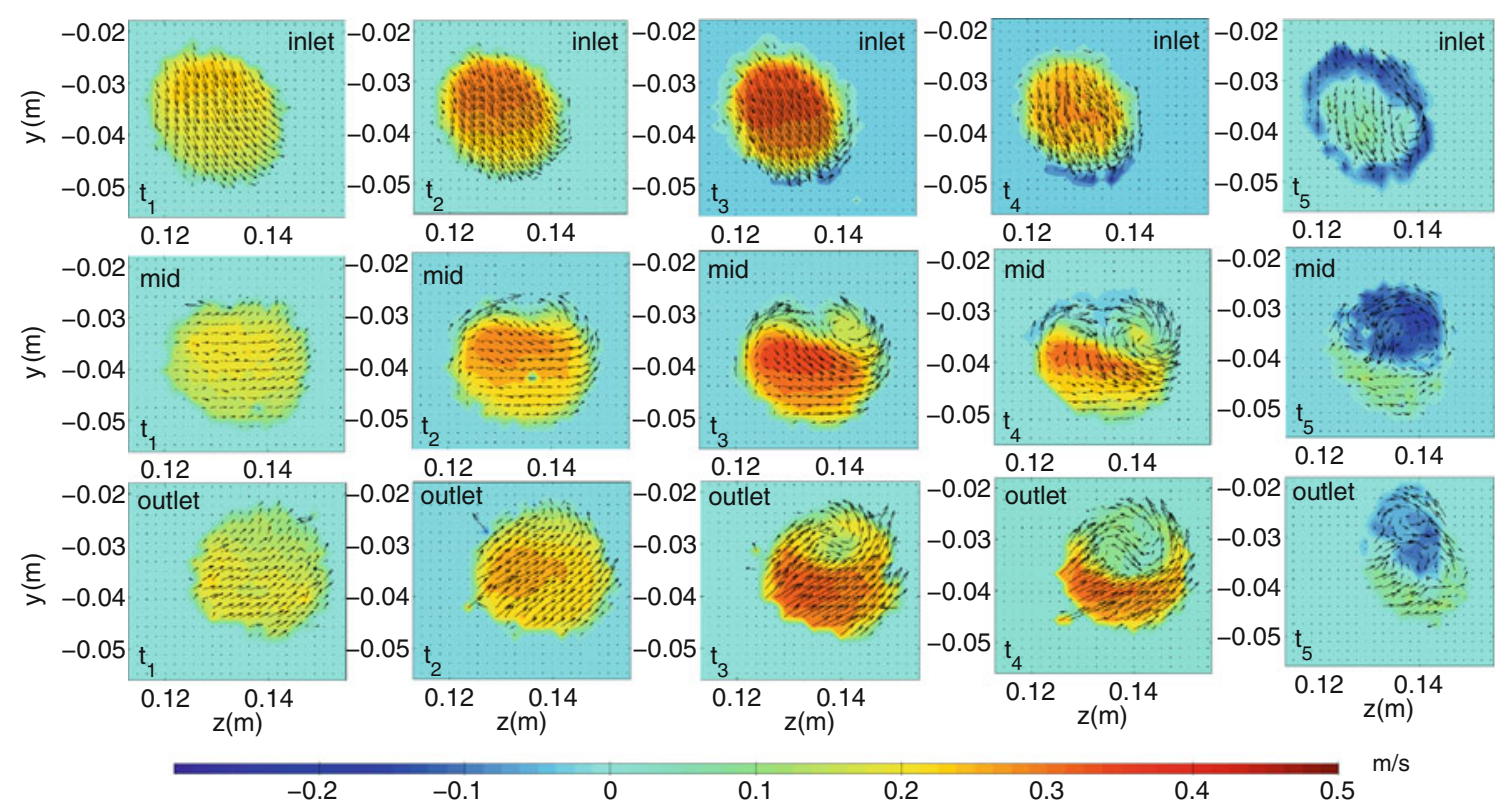

Fig. 11 Temporal evolution of the velocity distribution at different cross sections
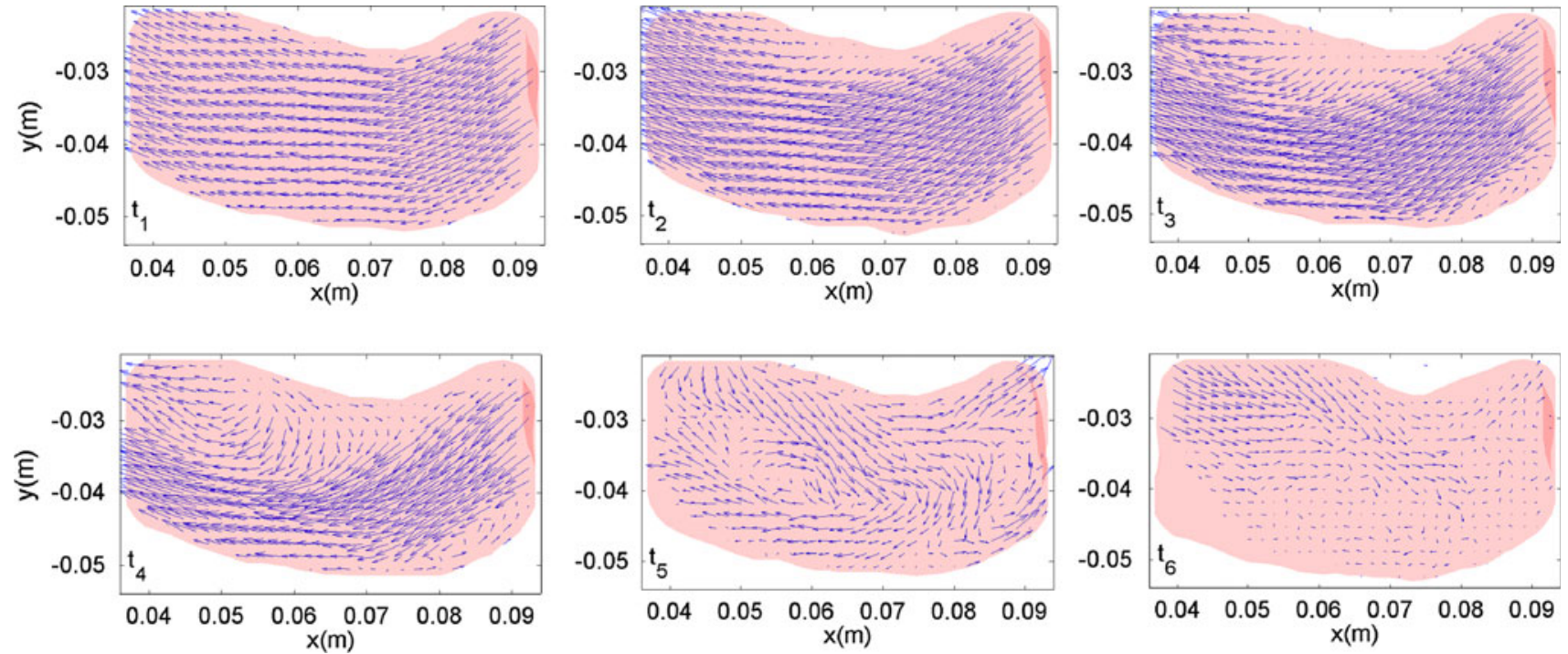

Fig. 12 Temporal evolution of the phase-averaged velocity vector map at mid-z plane

maps at $z=0.1361 \mathrm{~m}$, which corresponds to the mid-zplane of the measurement domain, are depicted in Fig. 12 for six different time instants. In the beginning of the systole $\left(t_{1}\right)$, the spatial distribution of the velocity field is relatively uniform. At the second time instant $\left(t_{2}\right)$, the flow velocities are mainly oriented along the axial direction, and the spatial pattern of the streamwise velocity field is similar to the prior time instant. At the peak systole $\left(t_{3}\right)$, a separation zone starts developing along the inner aortic wall. In the deceleration phase $\left(t_{4}\right)$, the separation zone becomes more distinct and larger rotational patterns occur distant from the inlet cross section. In the beginning of the diastolic phase $\left(t_{5}\right)$, the dominance of the retrograde flow becomes apparent. The spatial distribution of the streamwise velocity field transforms into an irregular pattern. At the inlet section, a retrograde flow region develops along the inner and outer walls. The spatial pattern of the velocity field varies downstream. The reversal flow develops along the inner wall of the region distal to the inlet cross section, whereas the antegrade flow is more dominant along the outer wall distal to the inlet cross section. At the final time instant $\left(t_{6}\right)$, the magnitudes of the streamwise velocities 

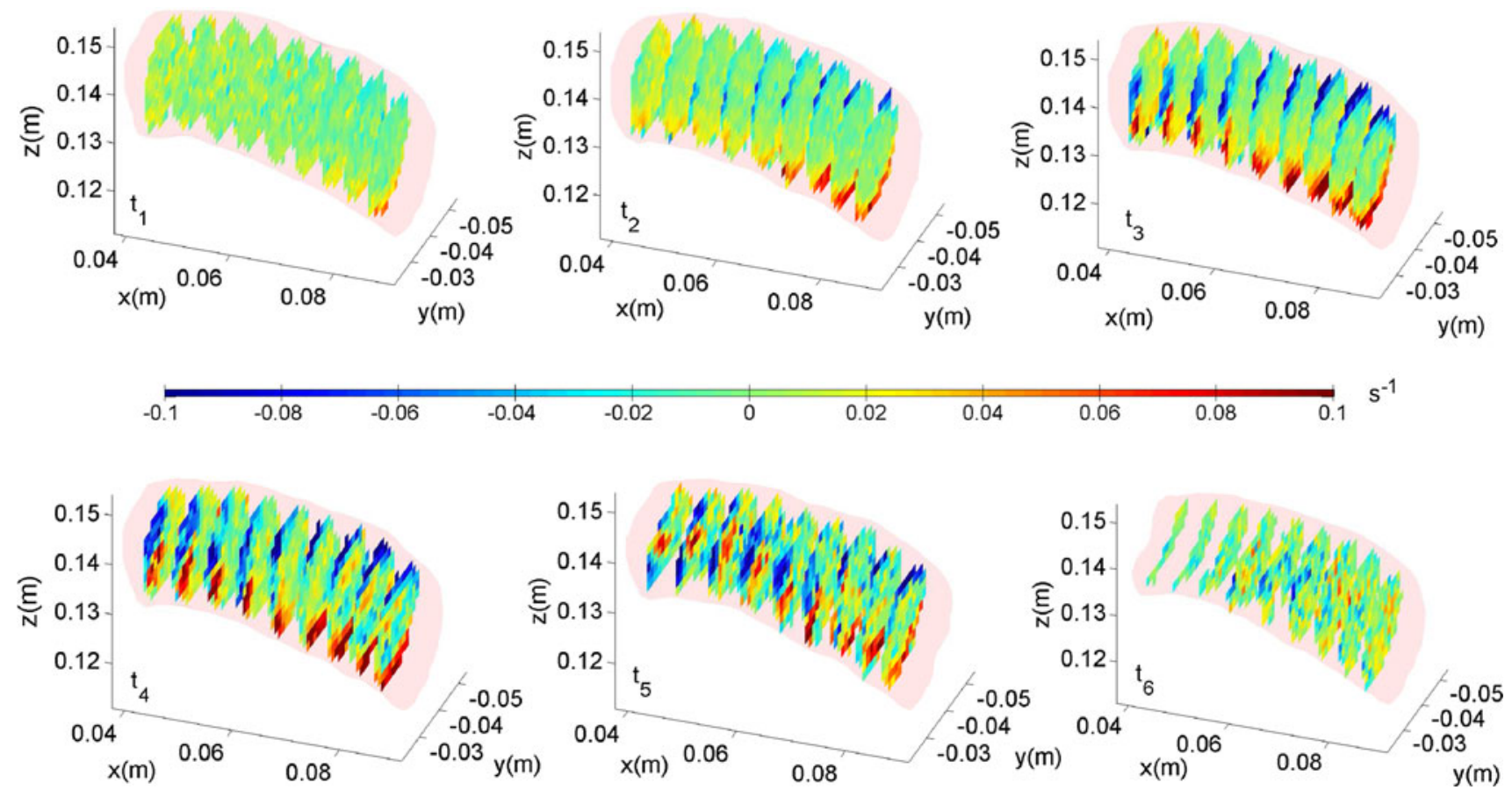

Fig. 13 Temporal evolution of streamwise vorticity $\left(\omega_{x}\right)$ (The blue coded vorticity field corresponds to the clockwise streamwise vorticity and the red coded vorticity field corresponds to the anticlockwise streamwise vorticity)

decay over the entire domain. The effect of the bidirectional flow can still be seen distal to the inlet cross section.

From the analysis of the phase-averaged velocity field, it is observed that rotational flow regions and counter-rotating vortices develop along the pulse. In Fig. 13, the streamwise vorticity component $\left(\omega_{x}\right)$ at six different time instants is depicted. In the beginning of the systole $\left(t_{1}\right)$, the streamwise vorticity field is relatively uniform for all cross sections. There is no distinct pattern of rotational zones occurring at this time instant. In the second time instant $\left(t_{2}\right)$, counter-clockwise streamwise vorticity develops along the anterior wall of the ascending aorta and clockwise streamwise vorticity at the posterior wall. In the peak systole $\left(t_{3}\right)$, the counter-clockwise streamwise vortices develop at the inner wall distal to the inlet cross section, whereas the clockwise streamwise vortices develop along the posterior wall of the aorta in the proximity of the inlet cross section. The clockwise streamwise vorticity pattern is shifted toward the inner wall of the aorta distal to the inlet cross section. In the deceleration phase $\left(t_{4}\right)$, the counterrotating vortices are located at the inner wall of the aorta distal to the inlet cross section. In the beginning of the diastolic phase $\left(t_{5}\right)$, the spatial distribution of the vorticity field becomes more disorganized compared to the systolic phase. Distal to the inlet cross section, the location of the counter-rotating vortices is shifted as a consequence of the reversal flow. At the final time instant $\left(t_{6}\right)$, the counterrotating vorticity regions vanish and the vorticity magnitudes attenuate considerably.
The vorticity field analysis shows that the flow in the ascending aorta has significant streamwise vorticity at certain time instants. The temporal and spatial evolutions of the velocity and vorticity field allow to investigate the helicity of the flow. In this study, a general qualitative frame of the spiraling motion is presented. Lagrangian trajectories are used as the visual indicator of spiraling motion. Depending on the calibration and tracking quality, it is desirable to track longer trajectories. Since the aortic flow is a complex, three-dimensional and pulsatile flow, the tracking becomes more difficult. In this study, the average trajectory length is $0.016 \mathrm{~s}$ which corresponds to 112 frames. Lagrangian trajectories at three different time intervals are presented in Fig. 14. Blue-coded trajectories refer to antegrade flow and the red-coded trajectories refer to retrograde flow. In the first time instant $\left(t_{3}\right)$, the particles are tracked from $x=0.09 \mathrm{~m}$ and for a time interval of $0.1 \mathrm{~s}$ which corresponds to the period $\left(t_{3}, t_{3}+0.1\right)$. The spiraling motion originates from the outer wall in the proximity of the inlet cross section and evolves along the inner wall distal to the inlet cross section. In this time period, the antegrade flow is distinctively dominant over the entire domain. In the second time instant $\left(t_{4}\right)$, similar to the prior time instant, the particles are tracked from $x=0.09 \mathrm{~m}$ and for a time interval of $0.1 \mathrm{~s}$ which corresponds to the period $\left(t_{4}, t_{4}+0.1\right)$. The helical motion of the flow is preserved over the entire domain. It is visible that the retrograde flow develops along the inner wall of the ascending aorta, whereas the antegrade flow is substantially 

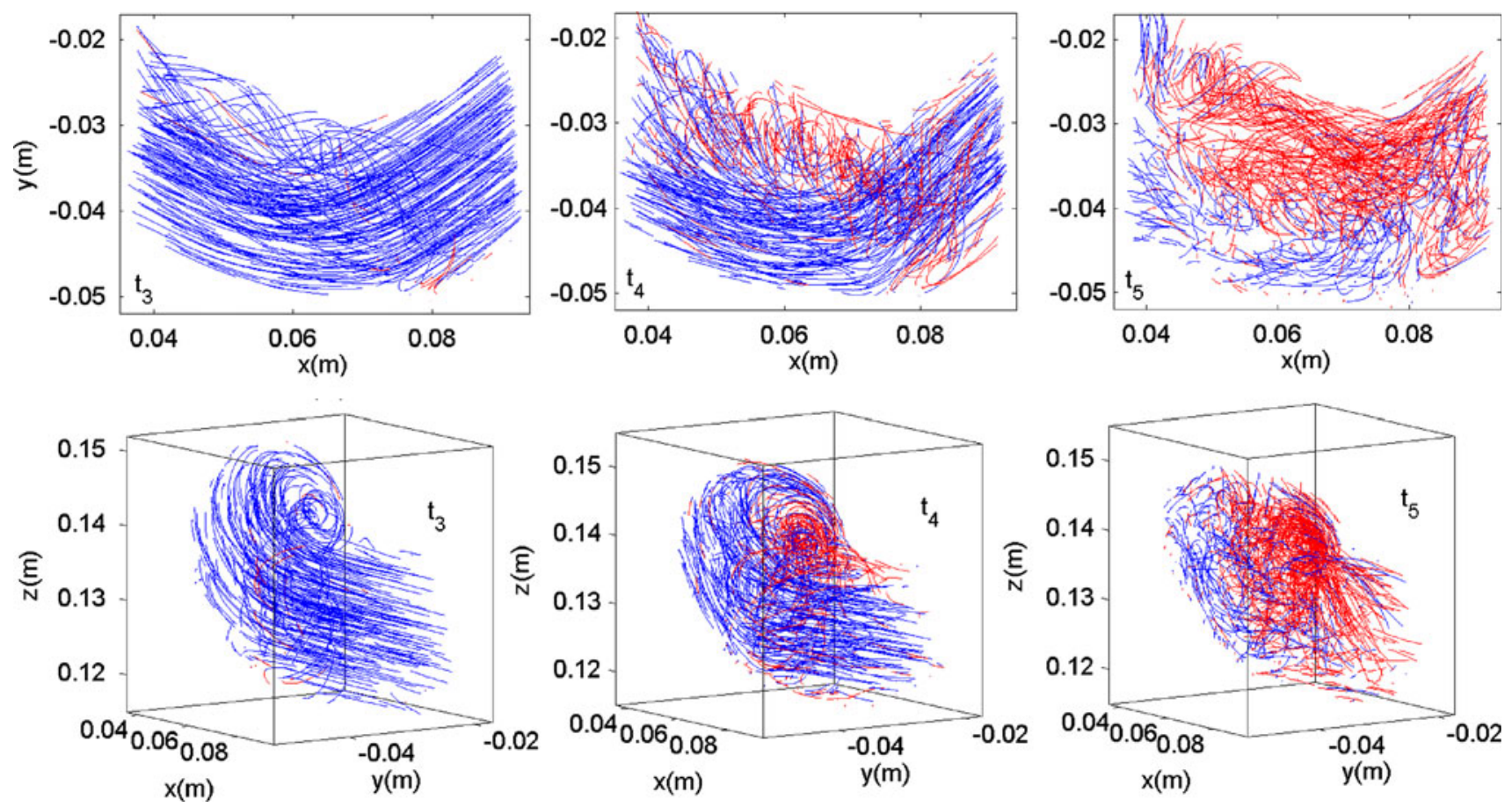

Fig. 14 Lagrangian trajectories for three different time intervals

located along the outer wall. In the final time instant $\left(t_{5}\right)$, the particles are tracked from $x=0.09 \mathrm{~m}$ and over a time interval of $0.1 \mathrm{~s}$ which corresponds to the period $\left[t_{5}\right.$, $\left.t_{5}+0.1\right]$. It is seen from the figure that the spiraling motion vanishes and the flow transforms into a more disorganized pattern. The dominance of the retrograde flow becomes more significant over the entire investigation volume with the exception of the outer wall.

In the cardiovascular system, the elevated turbulent fluctuations affect the transport efficiency. In atherosclerosis, low and oscillating shear stresses, flow separation, non-laminar blood flow patterns and turbulence have been suggested as possible atherogenic factors (Moore et al. 1994).

3D-PTV provides both Lagrangian and Eulerian information which helps to assess the disturbed flow. The comparison of mean and turbulent kinetic energies, averaged over the entire investigation domain, as a function of time is depicted in Fig. 15. The investigation domain is divided into boundary and bulk regions, each representing $50 \%$ of the cross-sectional area. It can be seen from the figure that the variations of the mean and turbulent kinetic energies are different. In the ascending aorta, the mean kinetic energy curve follows the volumetric flux curve through the systolic phase in Fig. 9. There is a distinct behavior of turbulent kinetic energies at the boundary and in the bulk. In the boundary region, the turbulent kinetic energy is higher in the early systole and reaches a maximum at the peak systole qualitatively similar to the mean

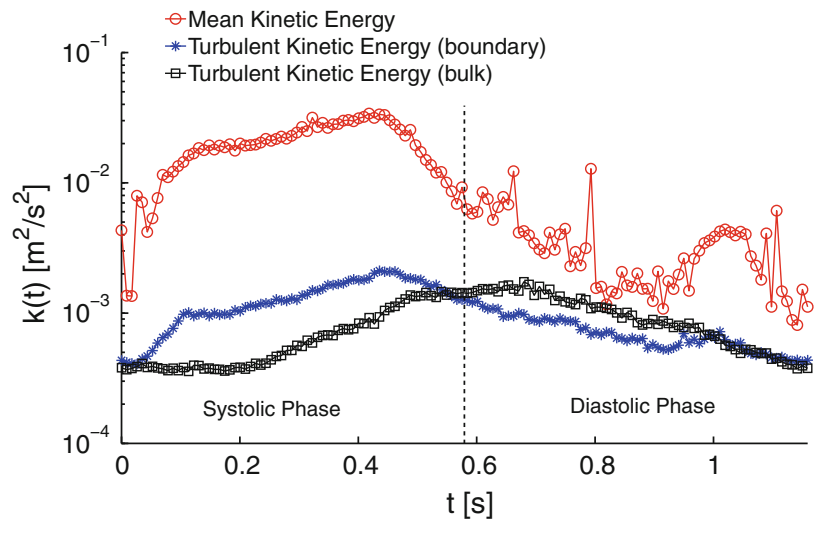

Fig. 15 Variation of mean kinetic energy and turbulent kinetic energy over a pulse

kinetic energy curve. Thereafter, a significant decay of the turbulent kinetic energy occurs in the deceleration phase. Unlike the boundary turbulent kinetic energy, the bulk turbulent kinetic energy is lower in the early systole. Thereafter, there is a slight increase up to the mid systole. The curve reaches a maximum later than in the case of the boundary kinetic energy. The discrepancy between the boundary and bulk turbulent kinetic energies is more apparent in the systolic phase, whereas toward the end of diastole, the two quantities are very close to each other. The magnitude of the bulk turbulent kinetic energy is higher than that of the boundary kinetic energy during the diastole. 
Figure 16 displays the contours of time-averaged mean and turbulent kinetic energies in $x y$ - and $y z$-planes. The cross sections $x 1, x 2$ and $x 3$ refer to the cross sections I, II and III depicted in Fig. 6. There is an apparent difference in the spatial distribution of both mean and turbulent kinetic energies. Analyzing the spatial distribution of the mean kinetic energy (MKE) in the $x y$-plane shows that the mean kinetic energy is higher in the bulk than at the walls. Distal from the inlet cross section the region of high MKE reaches the outer wall. At the inlet cross section, it is higher at the inner wall, consistent with the behavior of the streamwise velocity shown above. The anterior and posterior walls of the aorta exhibit comparatively higher values of mean kinetic energy. The spatial distribution of the turbulent kinetic energy (TKE) is distinctively different from the spatial distribution of the mean kinetic energy. The spatial distribution of the time-averaged turbulent kinetic energy in the $x y$-plane shows significant TKE in the bulk region away from the inlet. Also, close the boundaries TKE reaches fairly high values all along the $x y$-plane. At the inlet cross section (section $x 1$ in Fig. 16 bottom), TKE is high close to the walls. This is possibly due to the fact that the inflow velocity profile is relatively plug-like (Fig. 10), and strong shear exists close to the walls. For the mid and outlet cross sections ( $x 2$ and $x 3$ in Fig. 16 bottom) the time-averaged TKE is high in the bulk, which can be mainly associated with the disordered flow at diastole (Fig. 15), whereas the high TKE regions at the walls originate mostly in the systolic phase.
Figure 17 shows the spatio-temporal evolution of the root-mean-square fluctuation components at mid $z$-plane and at the three different cross sections depicted in Fig. 6. It is seen that the temporal evolution of the streamwise fluctuation component varies downstream, whereas the other two fluctuation components $\left(v_{\mathrm{rms}}^{\prime}, w_{\mathrm{rms}}^{\prime}\right)$ have similar temporal evolution for all cross sections. At the inlet section, streamwise fluctuations $\left(u_{\mathrm{rms}}^{\prime}\right)$ are high along the boundaries in the systolic phase. In the deceleration phase, the high streamwise fluctuation zone is found in the bulk region and decays toward the end of the pulse (Fig. 17 top, left). At the mid-cross section, the pattern of high $u_{\mathrm{rms}}^{\prime}$ has some resemblance with that of the inlet cross section, but during systole, it is comparatively weaker at the walls and high $u_{\mathrm{rms}}^{\prime}$ in the bulk occur earlier in time as one moves downstream. Different from the streamwise velocity fluctuations, the in-plane components show weak fluctuations during most of systole (Fig. 17 center and right). They become appreciable only in the deceleration phase. At the mid-cross section, strong fluctuations exist until the end of the pulse both for $v_{\mathrm{rms}}^{\prime}$ and $w_{\mathrm{rms}}^{\prime}$, whereas at the inlet and outlet cross sections, they seem to decay earlier. The results presented in Fig. 17 are consistent with the spatially averaged evolution shown above (Fig. 15). It is clearly seen that the fluctuation components contributing to TKE are higher along the boundaries in the systolic phase. In the diastolic phase, the high fluctuation region develops in the bulk region for all fluctuation components.
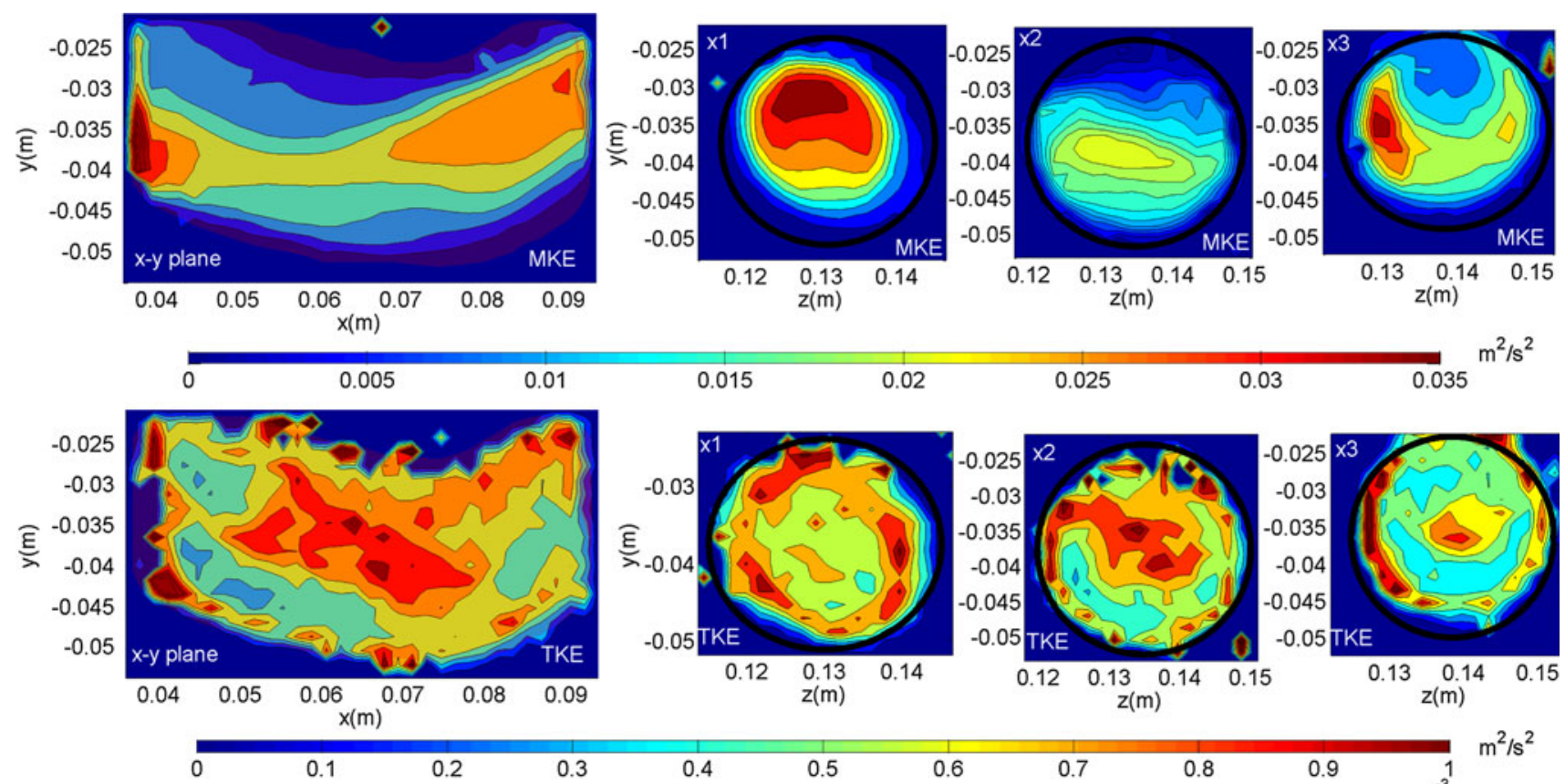

$0.015 \quad 0.02$

$0.025 \quad 0.03$
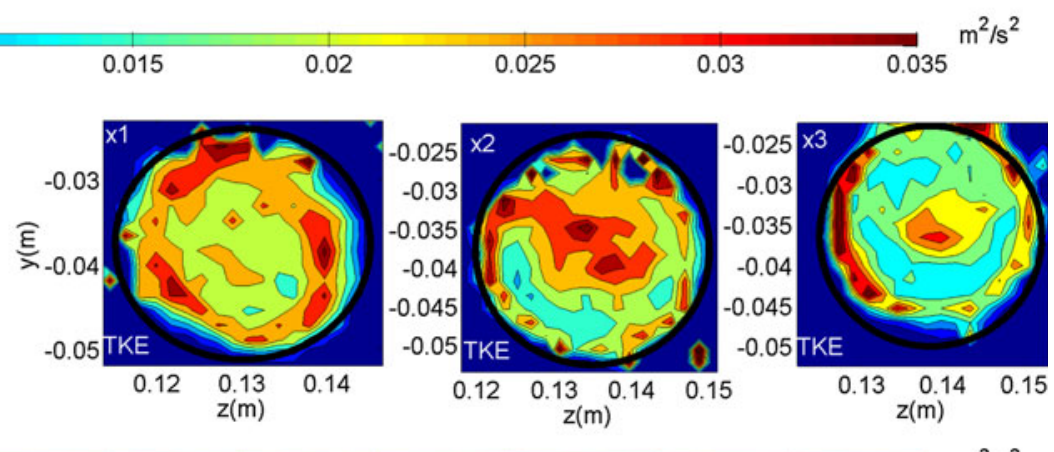

$3 \quad 0.4$

0.5

0.6

0.7

0.8

0.9

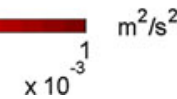

Fig. 16 Contours of time-averaged mean and turbulent kinetic energies in $x y$-plane and $y z$-plane 

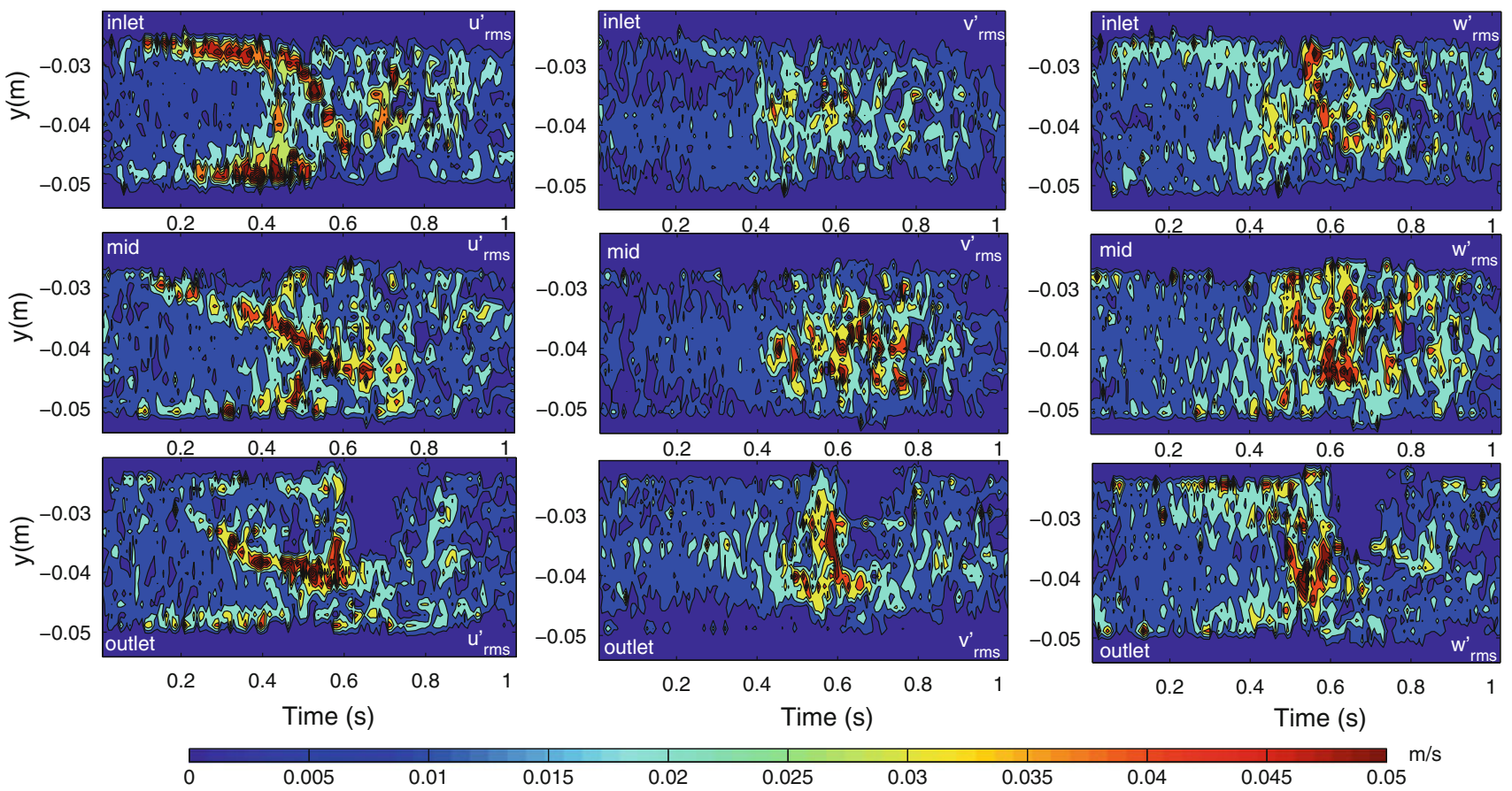

Fig. 17 Spatio-temporal evolution of the $u_{\mathrm{rms}}^{\prime}, v_{\mathrm{rms}}^{\prime}$ and $w_{\mathrm{rms}}^{\prime}$

In the presented setup, it is difficult to assess the rate of dissipation directly with 3D-PTV as it would require to resolve the velocity gradients. In the following, we use single-particle dispersion as a surrogate for dissipation measurements. This is justified as particle dispersion in an average sense is directly related to the rate of dissipation, as $r^{2} \sim \varepsilon^{n}$, with $n=2 / 3 \ldots 1$, depending on the precise conditions. It is possible to access particle dispersion characteristics by using Lagrangian information obtained by Particle Tracking Velocimetry. By means of turbulent kinetic energy, Lagrangian trajectories and particle dispersion, a general indication of the existence of turbulence can be obtained. As a final Lagrangian result, we present measurements of single-particle dispersion at various times during one pulse cycle. These results demonstrate the non-negligible dispersive, and thus mixing, character of the pulsatile aortic flow, which peaks in the early phase of diastole. To estimate single-particle dispersion in our flow which has a nonzero mean and which is pulsatile, we study the evolution of the separation vector $\mathbf{r}(\tau)$ between the position of real flow tracers $\mathbf{x}_{i}\left(t_{0}+\tau\right)$ and the position $\mathbf{x}_{m}\left(t_{0}+\tau\right)$ of a virtual tracer that is released at
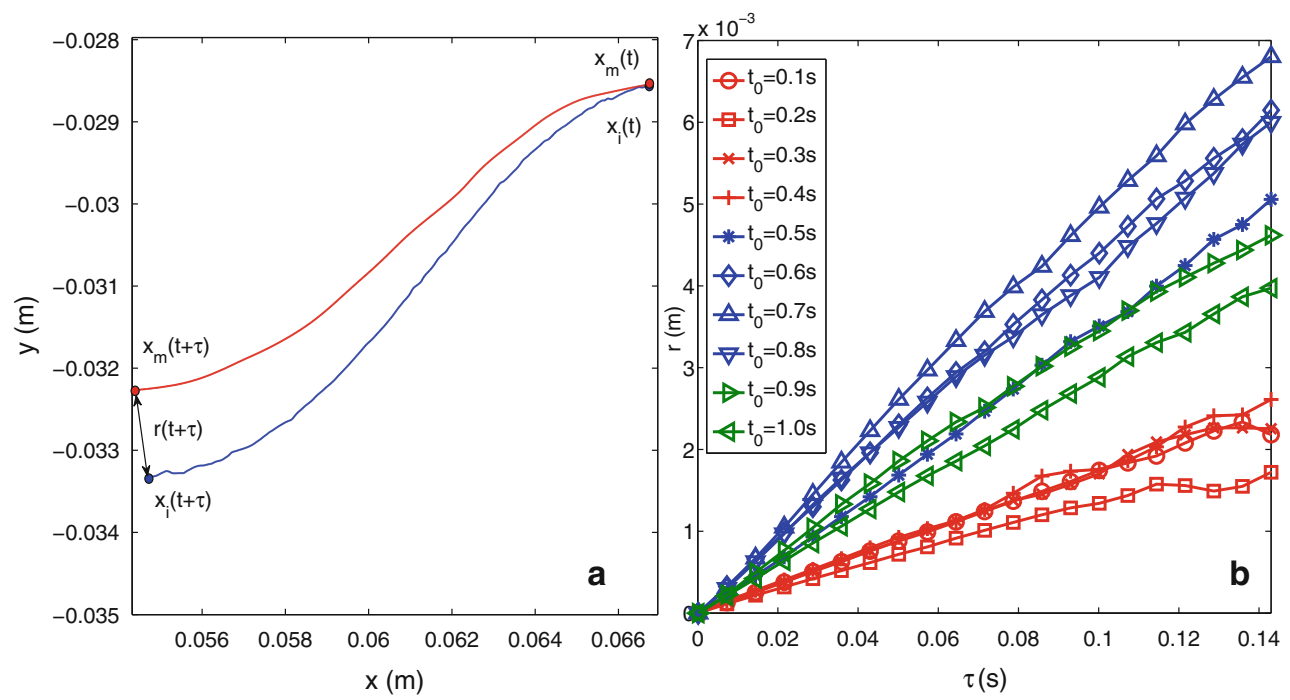

Fig. 18 Variation of particle separation over time lag 
the same $\mathbf{x}_{i}\left(t_{0}\right)$ but which is advected with the mean flow field, as it is sketched in Fig. 18a. The presented dispersion analysis uses the information of particle positions that are passing through the mid-cross section for various times during the pulse. For each real position in the mid-cross section, we release a mean particle that is advected by the mean phase-averaged flow. Separation statistics were collected for ten different release times, $t_{0}$, spread over the entire pulse cycle. From Fig. 18b, it is apparent that systolic dispersion is fairly small, but nonzero. Toward diastole and in the first half of the diastole, dispersion grows to a maximum that peaks at $t_{0}=0.7 \mathrm{~s}$, which coincides with the peak time of TKE shown above in Fig. 15. In the late phase of diastole, dispersion again decreases, due to decreasing velocity and despite the still turbulent nature of the flow. The strong diastolic dispersion is pointing toward genuine turbulence dynamics, within each pulse. The peak separation velocity is determined to be of significant magnitude as $\Delta u_{\text {peak }} \sim 0.05 \mathrm{~m} \mathrm{~s}^{-1}$, which is $\sim 10 \%$ of the mean peak flow velocity. Regardless whether the flow can be said to be truly turbulent or not it seems that the pulsatility definitely triggers a dispersive property of the aortic flow.

\section{Conclusion}

In this study, an in vitro analysis of the aortic flow in a healthy and compliant aortic phantom is presented. The velocity profile at the inlet of the ascending aorta is relatively flat with a skew toward the inner aortic wall in the early systole which agrees with the results of Chadran et al. (1993) and Naruse and Tanishita (1996). The flow field analysis shows that in the diastolic phase, bidirectional flow is observed with a pronounced retrograde flow developing along the inner aortic wall, whereas the antegrade flow region migrates toward the outer wall of the aorta. The temporal and spatial distribution of the retrograde flow shows a good agreement with the studies of Chadran et al. (1993), Segadal and Matre (1987), Morbiducci et al. (2011) and Kilner et al. (1993). The spiraling pattern of the flow is substantially affected by the retrograde flow. In the deceleration phase, counter rotational vortices develop along the inner curvature of the aorta. The helical pattern is indistinct in the systolic phase, whereas a coherent bi-helical structure develops in the deceleration phase which is consistent with the results of Morbiducci et al. (2011). Kilner et al. (1993) showed that the helical flow develops along the inner curvature as the peak velocity region migrates outward, and this region is replaced by the retrograde flow which shows great similarity to the results presented in this study.
The temporal and spatial distributions of the turbulent fluctuations show that there is an apparent difference between the bulk and boundary turbulent kinetic energies along the ascending aorta. The boundary kinetic energy is higher during the systolic phase and follows the characteristics of the volumetric flux curve. In the deceleration phase and diastolic phase, the boundary turbulent kinetic energy decays. Contrarily, the bulk turbulent kinetic energy is lower in the early systole and increases, starting from the mid systole. A peak turbulent kinetic energy region is observed in the early diastole whereafter the magnitude of the turbulent kinetic energy attenuates through the diastolic phase. It can be said that the temporal evolution of the bulk turbulent kinetic energy shows good agreement with the results of Yamaguchi et al. (1987). They concluded that the turbulence intensity increases in the peak systole and reaches a maximum value in the mid deceleration phase, whereas the turbulent intensity decays during the rest of the diastolic phase.

To investigate the existence of turbulence, particle dispersion extracted from Lagrangian information is used. The picture that arises from a single-particle dispersion analysis is consistent with our results on the temporal evolution of the field of turbulent fluctuations. It is determined that the particle dispersion is comparatively smaller in the systolic phase. There is a significant increase in the late systole and early diastole, and a maximum is reached in mid-diastolic phase which supports the temporal evolution analysis of the turbulent fluctuations.

The experiments confirm that the aorta model mimics the real aortic flow closely. This allows to look into specific questions in further steps. These questions are related among other things to the performance of artificial heart valves, influence of pathological flow conditions on the onset of turbulent flow or the comparison with medical imaging methods.

Acknowledgments This work was supported by ETH Research Grant ETH-24 08-2. Support from COST Action MP0806 is kindly acknowledged.

\section{References}

Balducci A, Grigioni M, Querzoli G, Romano GP, Aniele CD, D'Avenio G, Barbaro V (2004) Investigation of the flow field downstream of an artificial heart valve by means of PIV and PTV. Exp Fluids 36:204-213

Belz GG (1995) Elastic properties and Windkessel function of the human aorta. Cardiovasc Drugs Ther 9(1):73-83

Blake J, Easson W, Hoskins P (2009) A dualphantom system for validation of velocity measurements in stenosis model under steady flow. Ultrasound Med Biol 35(9):1510-1524

Bogren H, Buonocore M, Valente R (2004) Four dimensional magnetic resonance velocity mapping of blood flow patterns in the aorta in patients with atherosclerotic coronary artery disease 
compared age-matched normal subjects. J Magn Reson Imaging 19(4):417-427

Boutsianis E, Guala M, Olgac U, Wildermuth S, Hoyer K, Ventikos Y, Poulikakos D (2009) CFD and PTV steady flow investigation in an anatomically accurate abdominal aortic aneurysm. J Biomech Eng 131(1):011008-011015

Chandran KB (1993) Flow dynamics in the human aorta. J Biomed Eng 115:611-616

Dyverfeldt P, Sigfridsson A, Kvitting J, Ebbers T (2006) Quantification of intravoxel velocity standard deviation and turbulence intensity by generalizing phase-contrast mri. Magn Reson Med 56(4):850-858

Dyverfeldt P, Kvitting J, Sigfridsson A, Engvall J, Bolger A, Ebbers T (2008) Assessment of fluctuating velocities in disturbed cardiovascular blood flow : in vivo feasibility of generalized phasecontrast mri. J Magn Reson Imaging 28(3):655-663

Frydrychowicz A, Harloff A, Zaitsev BJM, Weigang E, Bley T, Langer M, Hennig J, Markl M (2007) Time resolved, 3-dimensional magnetic resonance flow analysis at $3 \mathrm{t}$ : visualization of normal and pathological aortic vascular hemodynamics. J Comput Assist Tomogr 31(1):9-15

Holzner M, Liberzon A, Nikitin N, Luthi B, Kinzelbach W, Tsinober A (2008) A lagrangian investigation of the small-scale features of turbulent entrainment through particle tracking and direct numerical simulation. J Fluid Mech 598:465-475

Hoyer K, Holzner M, Luthi B, Guala M, Liberzon A, Kinzelbach W (2005) 3d scanning particle tracking velocimetry. Exp Fluids 39:923-934

Kasagi N, Matsunaga A (1995) Three-dimensional particle tracking velocimetry measurement of turbulence statistics and energy budget in a backward-facing step flow. Int J Heat Fluid Flow 16(6):477-485

Kilner P, Yang G, Mohiaddin R, Firmin D, Longmore D (1993) Helical and retrograde secondary flow patterns in the aortic arch studied by three-directional magnetic resonance velocity mapping. Circulation 88:2235-2247

Kozerke S, Hasenkam J, Pedersen E, Boesiger P (2001) Visualization of flow patterns distal to aortic valve prostheses in humans using a fast approach for cine $3 \mathrm{~d}$ velocity mapping. J Magn Reson Imaging 13(5):690-698

$\mathrm{Ku}$ D (1997) Blood flow in arteries. Annu Rev Fluid Mech 29:399-434

Kvitting J, Dyverfeldt P, Sigfridsson A, Frazen S, Wigstrom L, Bolger A, Ebbers T (2010) In vitro assessment of flow patterns and turbulence intensity in prosthetic heart valves using generalized phase-contrast mri. J Magn Reson Imaging 31(5): 1075-1080

Lüthi B, Tsinober A, Kinzelbach W (2005) Lagrangian measurement of vorticity dynamics in turbulent flow. J Fluid Mech 528: $87-118$

Maas H, Gruen A, Papantoniou D (1993) Particle tracking velocimetry in three-dimensional flows. Exp Fluids 15(2):133-146

Malik N, Dracos T, Papantoniou D (1993) Particle tracking velocimetry in three-dimensional flows. Exp Fluids 15(4-5):279-294
Markl M, Draney MT, Miller DC, Levin JM, Willamson EE, Pelc NJ, Liang DH, Herfkens RJ (2005) Time-resolved 3D magnetic resonance velocity mapping of aortic flow in normal volunteers and patients after valve-sparing aortic root replacement. J Thorac Cardiovasc Surg 130(2):456-463

McDonald D (1952) The occurence of turbulent flow in the rabbit aorta. J Physiol 118:340-347

Moore J, Xu C, Glagov S, Zarins C, Ku D (1994) Fluid wall shear stress measurements in a model of the human abdominal aorta:oscillatory behavior and relationship to atherosclerosis. Atherosclerosis 110:225-240

Morbiducci U, Ponzini R, Rizzo G, Cadioli M, Cobelli F, Maschito A, Montevecchi F, Redaelli A (2009) In vivo quantification of helical blood flow in human aorta by time resolved threedimensional cine phase contrast magnetic resonance imaging. Ann Biomed Eng 37(3):516-531

Morbiducci U, Ponzini R, Rizzo G, Cadioli M, Esposito A, Montevecchi F, Redaelli A (2011) Mechanistic insight into the physiological relevance of helical blood flow in the human aorta: an in vivo study. Biomech Model Mechanobiol 10:339-355

Naruse T, Tanishita K (1996) Large curvature effect on pulsatile entrance flow in a curved tube: model experiment simulating blood flow in an aortic arch. J Biomed Eng 118:180-186

Nerem RM, Wood NB, Seed WA (1972) An experimental study of the velocity distribution and transition to turbulence in the aorta. J Fluid Mech 52:137-160

Pedocchi F, Martin J, Garcia M (2008) Inexpensive fluorescent particles for large-scale experiments using particle image velocimetry. Exp Fluids 45:183-186

Schibli M, Wiesendanger M, Guzzella L, Hoyer K, Soellinger M, Kurtcuoglu V, Boesiger P (2008) In vitro measurement of ventricular cerebrospinal fluid flow using particle tracking velocimetry and magnetic resonance imaging. First Int Symp Appl Sci Biomed Commun Technol (ISABEL) 39:923-934

Segadal L, Matre K (1987) Blood velocity distribution in the human ascending aorta. Circulation 76(1):90-100

Stein P, Sabbah H (1976) Turbulent blood flow in the ascending aorta of humans with normal and diseased aortic valves. Circ Res 39:58-65

Stamatopoulos C, Mathioulakis DS, Papaharilaoub Y, Katsamouris A (2011) Experimental unsteady flow study in a patient-specific abdominal aortic aneurysm model. Exp Fluids 50:1695-1709

Virant M, Dracos T (1997) 3d ptv and its application on lagrangian motion. Meas Sci Technol 8(12):1539-1552

Wilneff J, Gruen A (2002) A new spatio-temporal matching algorithm for 3d-particle tracking velocimetry. The 9th international symposium on transport phenomena and dynamics of rotating machinery honolulu, Hawaii, USA

Wilson J, Cobb E, Kilpatrick F (1986) Fluorometric producers for dye tracing, vol 45. U.S. geological survey, techniques of water resources investigation, Book 3, U.S. Government printing office

Yamaguchi T, Kikkawa S, Parker K (1987) Simulation of nonstationary spectral analysis of turbulence in the aorta using a modified autoregressive or maximum entropy (ar/me) method. Med Biol Eng Comput 25:533-542 